\title{
Advanced Carbon Nanotubes Functionalization
}

\author{
A Setaro ${ }^{1}$ \\ ${ }^{1}$ Department of Physics, Freie Universität Berlin, ArnimalleE 14, 14195 Berlin \\ Email:setaro@physik.fu-berlin.de
}

\begin{abstract}
Similarly to graphene, carbon nanotubes are materials made of pure carbon in its $\mathrm{sp}^{2}$ form. Their extended conjugated $\pi$-network provides them with amazing quantum optoelectronic properties. Frustratingly, it also brings drawbacks: The $\pi-\pi$ stacking interaction makes as-produced tubes bundle together, blurring out all their quantum properties. Functionalization aims at modifying and protecting the tubes while hindering $\pi-\pi$ stacking. Several functionalization strategies have been developed to circumvent this limitation and for nanotubes applications to thrive. In this review, we summarize different approaches established so far, emphasizing the balance between functionalization efficacy and preservation of the tubes' properties. Major attention will be devoted to a functionalization strategy overcoming the covalent-noncovalent dichotomy and to the implementation of two advanced functionalization schemes: a) conjugation with molecular switches, to yield hybrid nanosystems with chemo-physical properties that can be tuned in a controlled and reversible way and b) plasmonic nanosystems, whose ability to concentrate and enhance the electromagnetic fields can be taken advantage of for enhancing the optical response of the tubes.
\end{abstract}

\section{Contents}

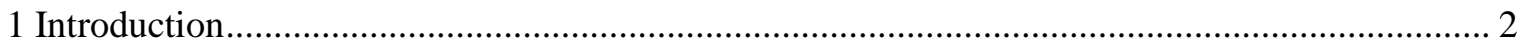

2 A brief primer on carbon nanotubes .......................................................................... 3

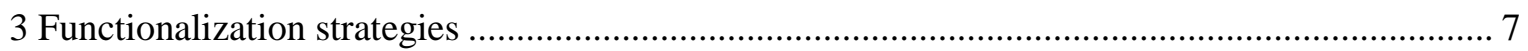

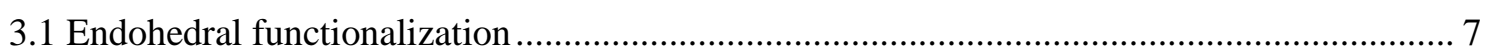

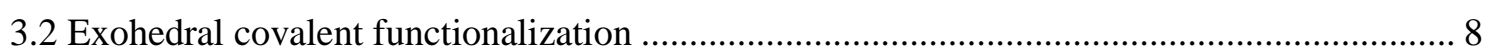

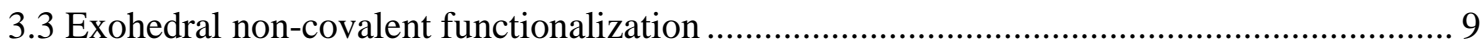

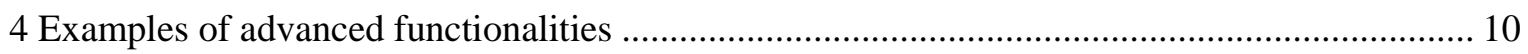

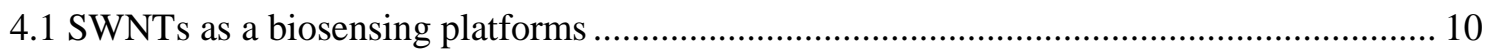

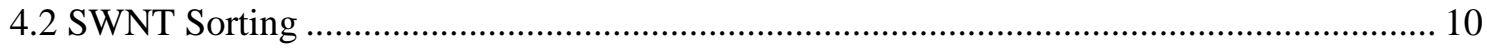

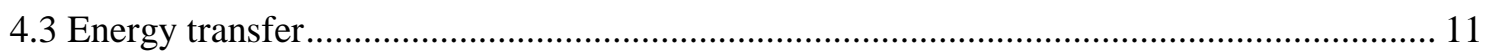

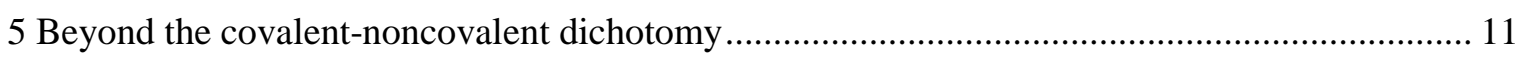

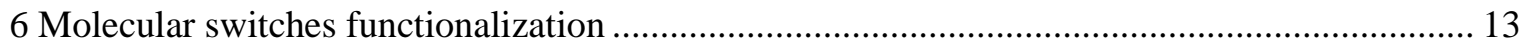

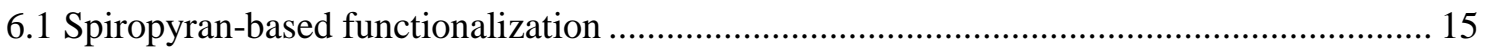

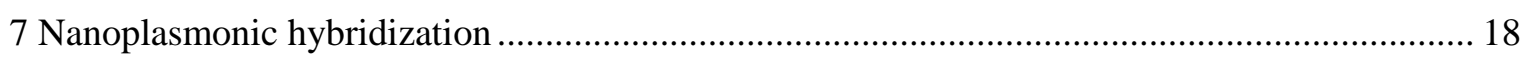

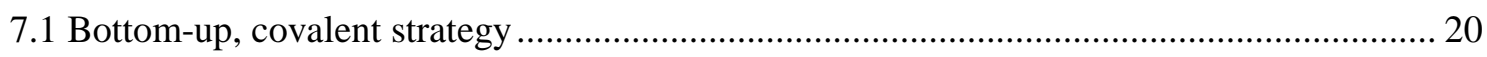

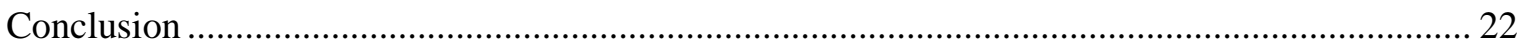

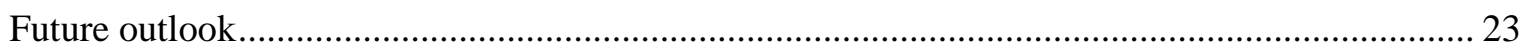

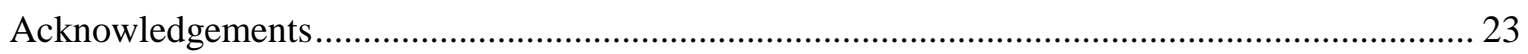

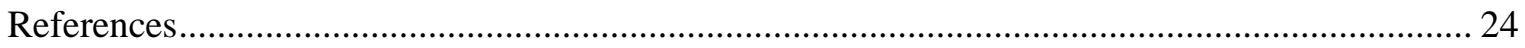




\section{Introduction}

Since their discovery [1], carbon nanotubes (CNTs) display outstanding properties, holding to the promise to innovate fundamental science and technological applications. They are cylinders with nanometric diameters that can extend up to the millimeter range, establishing a direct bridge between the nanoscopic and the macroscopic worlds. They are single conducting channel with ballistic transport capabilities and intrinsic mobilities exceeding common semiconducting systems [2] and promise to replace silicon for future generation electronics. Devices made out of suspended crossbar nanotubes have proven to lead to nonvolatile random-access memories [3]. They are more energy efficient then silicon-based devices by more than one order of magnitude and have been used to implement an all-nanotube universal functional computer running an operating system and performing several instructions in multi-task [4]. Their capability of building up bound electron pairs through excitonic rather than phononic coupling mechanisms promises to push superconductivity beyond the BCS landscape and design new exotic states of matter [5].

The in-plane carbon-carbon bond in $\mathrm{sp}^{2}$ carbon systems is one of the strongest bonds in nature. The exceptional mechanical stability and lightness of carbon nanotubes made them enter the collective imagery as the material for the space elevator [6]. Exploiting them as cantilever tips has made it possible to image single molecules by atomic force microscopy at room temperature [7]. Contrarily to other carbon allotropes, carbon nanotubes, as a direct consequence of their quantum nature, can exhibit semiconducting behavior and emit light. Their one-dimensional nature ensures efficient suppressed dielectric screening and makes their optical activity purely ruled by excitons stable at room temperature [8]. This pledges for advanced photonic and optoelectronic applications [9], such as room temperature single-photon sources [10], optical promotion of entanglement [11], and rectifying antennas to convert optical radiation directly into currents [12]. Their infrared emission lies in the second transparency window of tissue transparency and ensures large penetration with low scattering and autofluorescence [13]. This allows in vivo near-infrared imaging [14]. Their compatibility with biological environments makes them ideal for bio-applications: They have been proposed as drugdelivery nanocapsules [15] or to even selectively react to the acidity of the environment to carry and release drugs in the proximity of cancer cells [16].

Most of the nanotubes applications, anyway, rely on the tubes being isolated and in their pristine state. Unfortunately, as-produced nanotubes are far away from this requirement: They come strongly bundled together and comprising different kind of impurities resulting from the growth processes. Different strategies have been implemented to circumvent this obstacle and customize the tubes for targeted applications. After a brief introduction on CNTs in Section 2, some of the approaches developed so far will be highlighted in Section 3, emphasizing the implications of the delicate balance between strength of the functionalization and preservation of the properties of the tubes. Section 4 provides some examples of advanced functionalization schemes established so far. Section 5 is 
devoted to a novel approach that establishes a dogmatic change in the functionalization strategies, exploiting the strength of the covalent attachments while safeguarding the quantum optoelectronic properties of the tubes. After that, special attention will be devoted to two advanced functionalization examples to add new features and capabilities to the SWNTs: Conjugation with photochromic molecular switches, to tailor and control the properties of the tubes through exposure to radiation of the proper wavelength will be outlined in Section 6, while strategies for decoration of the tubes with nanoplasmonic particles to enhance their optical response will be reported in Section 7.

\section{A brief primer on carbon nanotubes}

CNTs are tubules made of pure carbon in its $\mathrm{sp}^{2}$ form. Similarly to graphene, the extended, conjugated skeleton network of in-plane covalently $\sigma$-bonded carbon atoms makes CNTs possess outstanding mechanical properties, being 100 times stronger than steel and with Young modulus that can reach 1 Tpa [17]. The optoelectronic features of the CNTs, moreover, are ruled by their delocalized $\pi$-electrons with their peculiar linear dispersion relation in the surrounding of the $\mathrm{K}$ points of the Brillouin zone [18]. While this renders graphene a semimetal, it makes carbon nanotubes display a way richer phenomenology, spanning from the metallic to the semiconducting behavior [19]. In this work, we will focus on single-walled carbon nanotubes (SWNTs), made out of a single cylinder. The most straightforward way to picture and easily predict the properties of a given SWNT is to depict it as a folded-up graphene sheet cut for a certain length and rolled up along a certain direction, both parameters specified through the chiral vector $\mathbf{c}=\left(\mathrm{n}_{1}, \mathrm{n}_{2}\right)$, expressed in terms of the base unit vectors of the graphene sheet. The so-called zone-folding approximation states that the properties of a specific SWNT can be predicted starting from the graphene dispersion relation, neglecting any curvature-induced changes and simply picking up the states corresponding to the electronic wavefunctions selected by the periodic boundary conditions dictated by c [17][18]. As a result of such selection process, SWNTs are metallic if $\bmod _{3}\left(\mathrm{n}_{1}-\mathrm{n}_{2}\right)=0$ and are semiconducting if $\bmod _{3}\left(n_{1}-n_{2}\right)=1,2$. The zone-folding predictions are valid as long as the $\sigma$ and $\pi$ orbitals of the tubes, which are orthogonal in the flat graphene, do not start mixing among each other. For very small nanotubes radii, indeed, the curved carbon structure allows the $\sigma$ and $\pi$ orbitals of the tubes to mix, yielding novel $\mathrm{sp}^{2} / \mathrm{sp}^{3}$ hybrid states that make the resulting tubes' behavior deviate from the zonefolding predictions [20]. The commercially available SWNTs used in optical experiments (e.g. HiPCOs and CoMoCATs) have diameters starting from $0.7 \mathrm{~nm}$, big enough to render such effects negligible. The $\mathrm{sp}^{2}$ hybridization state of the carbon network can thus be assumed to remain unaltered. The curvature, anyway, is the responsible for small degree of pyramidalization of the $\mathrm{C}$ atoms and misalignment of the $\pi$-orbitals between two neighboring $\mathrm{C}$ atoms, factors making carbon nanotubes chemically more reactive than graphene[21]. The resulting reactivity scales with inverse proportionality with respect to SWNTs' diameter [22],[23]. 
a)

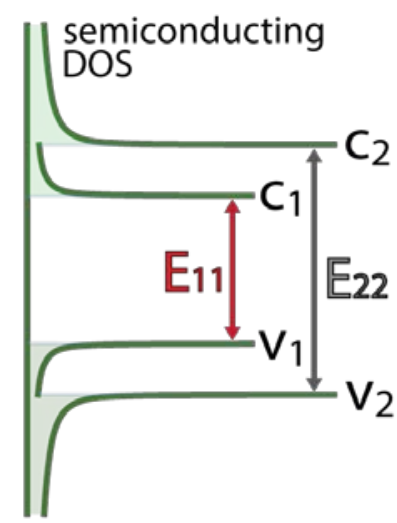

b)

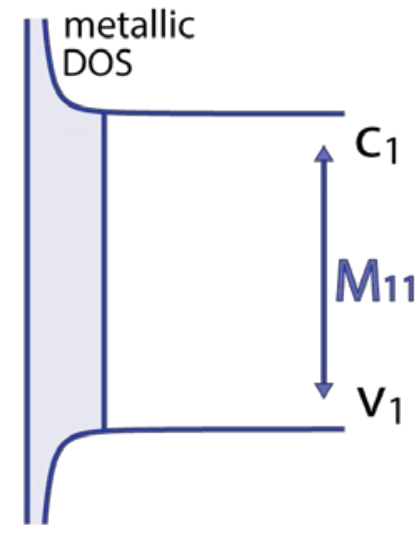

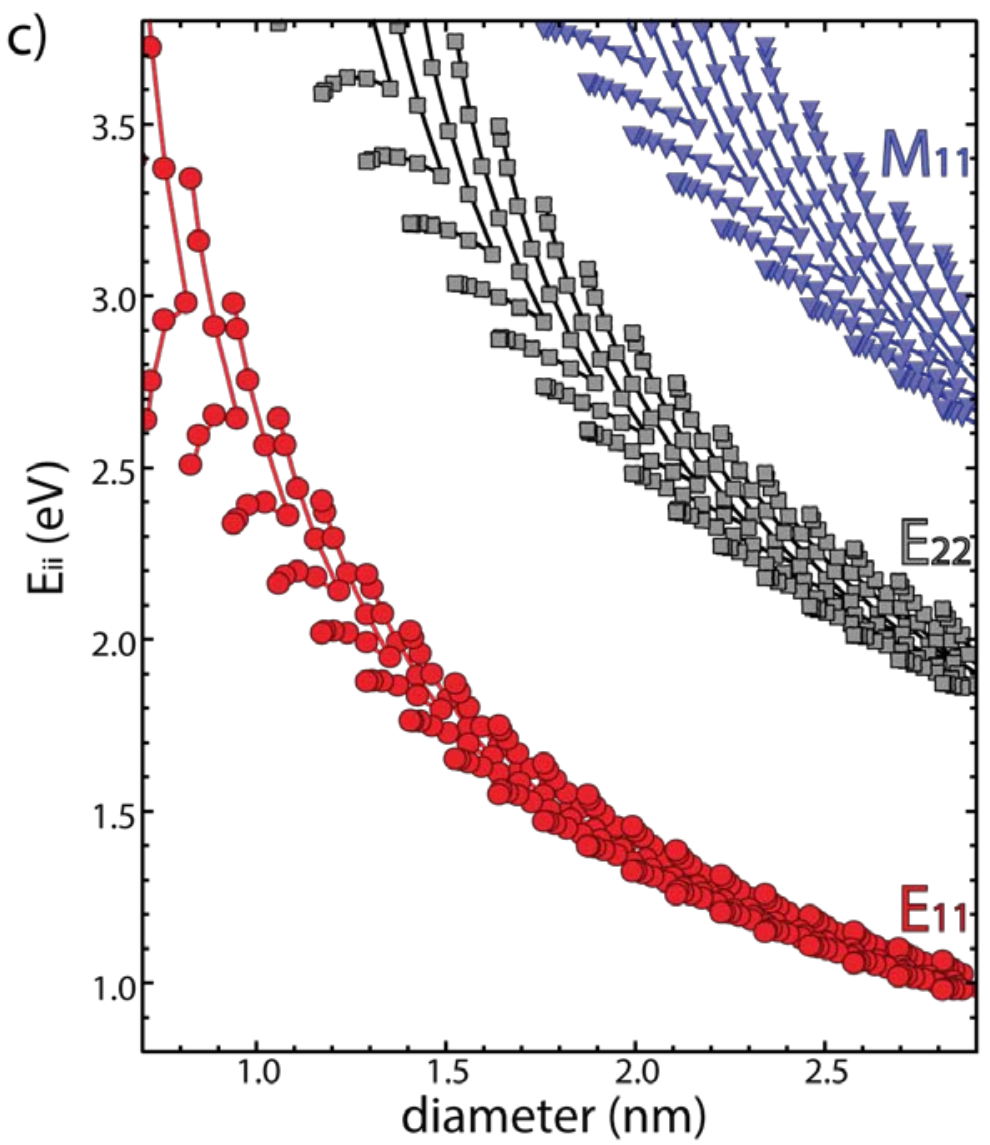

Figure 1: (a) Density of states of a semiconducting SWNT. The energy separation between the first van Hove singularities, respectively, in the conduction and valence band is denoted as $E_{11}$, while between the second is $E_{22}$ ( (b) Density of states of a metallic SWNT. The energy separation between the first van Hove singularities, respectively, in the conduction and valence band is denoted as $\mathbf{M}_{11}$. (c) Kataura plot showing the distribution of the transition energies for semiconducting $\left(\mathrm{E}_{11}\right.$ and $\left.\mathrm{E}_{22}\right)$ and metallic $\left(\mathrm{M}_{11}\right)$ [27].

The 1- $d$ nature of SWNTs reflects in their density of states with the characteristic sequence of van Hove singularities. The energetic position of such singularities is dictated by the linear dispersion relation of the $\pi$-electrons in the extended $\mathrm{sp}^{2}$ network, which makes the diameter dependence of transition energies of carbon nanotubes roughly scale as $d^{-1}$, with deviations from this trend, which are chirality dependent and are due to the trigonal warping effect [24],[25]. In 1999, H. Kataura introduced the plot, bearing his name, depicting the dependence of the transition energies of SWNTs towards their diameter [26]. By having a closer look at a Kataura plot, one can rationalize the deviations from the $d^{-1}$ trend of the transition energies by finding that the different branches are composed by nanotubes belonging to the so-called laola families, all characterized by possessing the same value of the family index $q=2 \mathrm{n}_{1}+\mathrm{n}_{2}$ [18]. Fig. 1 schematically depicts the density of states of (a) semiconducting and (b) metallic SWNTs. Panel (c) shows the Kataura plot for the first two transition energies for semiconducting tubes (labeled, respectively, as $E_{11}$ and $E_{22}$ ) and the first transition energies for the metallic tubes (labeled as $\mathrm{M}_{11}$ ). We will see later that the characteristic patterning of the nanotubes energetics is a valid reference tool to straightforwardly identify and analyze them experimentally. 
Several linear and nonlinear optical processes take place within the tubes and can be exploited to characterize them. Among the linear ones, the most commonly used are the absorption spectroscopy and the two-dimensional excitation-emission spectroscopy, known also as excitation photoluminescence spectroscopy (PLE) [9],[28]-[31]. Among the nonlinear optical spectroscopies, the most powerful technique to gain information about fundamental properties of the nanotubes is Raman spectroscopy. SWNTs have several Raman-active modes, some originating from first-order transition processes, like the $\mathrm{G}$ ( $1600 \mathrm{~cm}^{-1}$, due to the in-plane carbon vibrations) or the RBM (radial breathing modes, $150-300 \mathrm{~cm}^{-1}$, due to the tubes' expansion in the radial direction) modes, and some other arising from higher-order processes, such as the $\mathrm{D}\left(\sim 1600 \mathrm{~cm}^{-1}\right.$, activated by defects) and 2D ( $\sim 2600 \mathrm{~cm}^{-1}$, two-phonon process) bands. Their analysis can help identifying the tubes, gaining insight into their stress-strain state, changes in their Fermi level position, and so on. The ratio between the intensity of the D and G bands, moreover, is commonly used for estimating the defectivity of the carbon network. For an exhaustive review on the topic of Raman spectroscopy of carbon nanotubes, please refer to Ref. [32].

We will here mostly focus on the PLE, though, because it helps driving very direct assessment over the functionalization outcome: Beyond the identification of the SWNTs chirality, it most importantly ascertains whether the nanotubes are isolated or in bundles and if their $\pi$-conjugation is perturbed or not. The emission due to band-band recombination requires the excitation of the nanotube with photon energies resonating with the $\mathrm{E}_{22}$ transition between the second van Hove singularities in the valence and conduction band. After fast internal nonradiative thermalization processes, the electron and hole will recombine emitting a photon with the specific $E_{11}$ energy (process sketched in Fig. 2a). Other processes are suppressed by the selection rules [18]. It is also worth mentioning the socalled antenna effect, the suppression of the optical absorption perpendicular to the SWNT axis by depolarization [18]. The $\left(\mathrm{E}_{22}, \mathrm{E}_{11}\right)$ position of the emission spot in a PLE chart univocally identifies the associated nanotube species [33], for example the $(8,4)$ chiral species in Fig. 2b. Please note that several effects can induce small shifts in such positions, for example changes in the dielectric environment surrounding the tubes affect the dielectric screening within the tubes inducing the socalled solvatochromic shifts and reducing both values of the $E_{11}$ and $E_{22}$ transition values [34]. A different behavior is for example exhibited by SWNTs under strain, where the shifts of $E_{11}$ and $E_{22}$ are opposite [35]. Please note that, even if such effects shift the $\left(E_{22}, E_{11}\right)$ position, they do not alter the pattern of the laola branches, shifting the whole pattern without changing its structure [18]. Fig. 3c depicts this pattern in the pseudo-PLE chart containing the most common semiconducting SWNTs available in commerce for optical purposes. The nanotubes belonging to the same laola family are connected together for an easier visualization of the pattern. 
a)

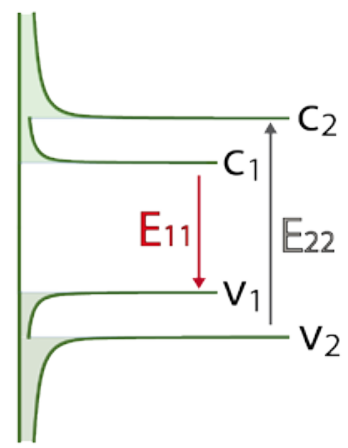

b)

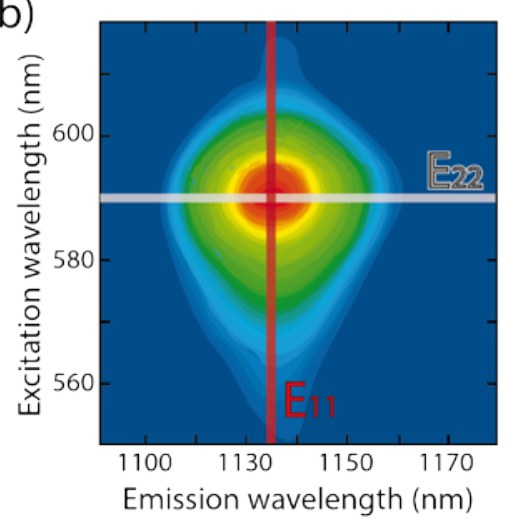

c)

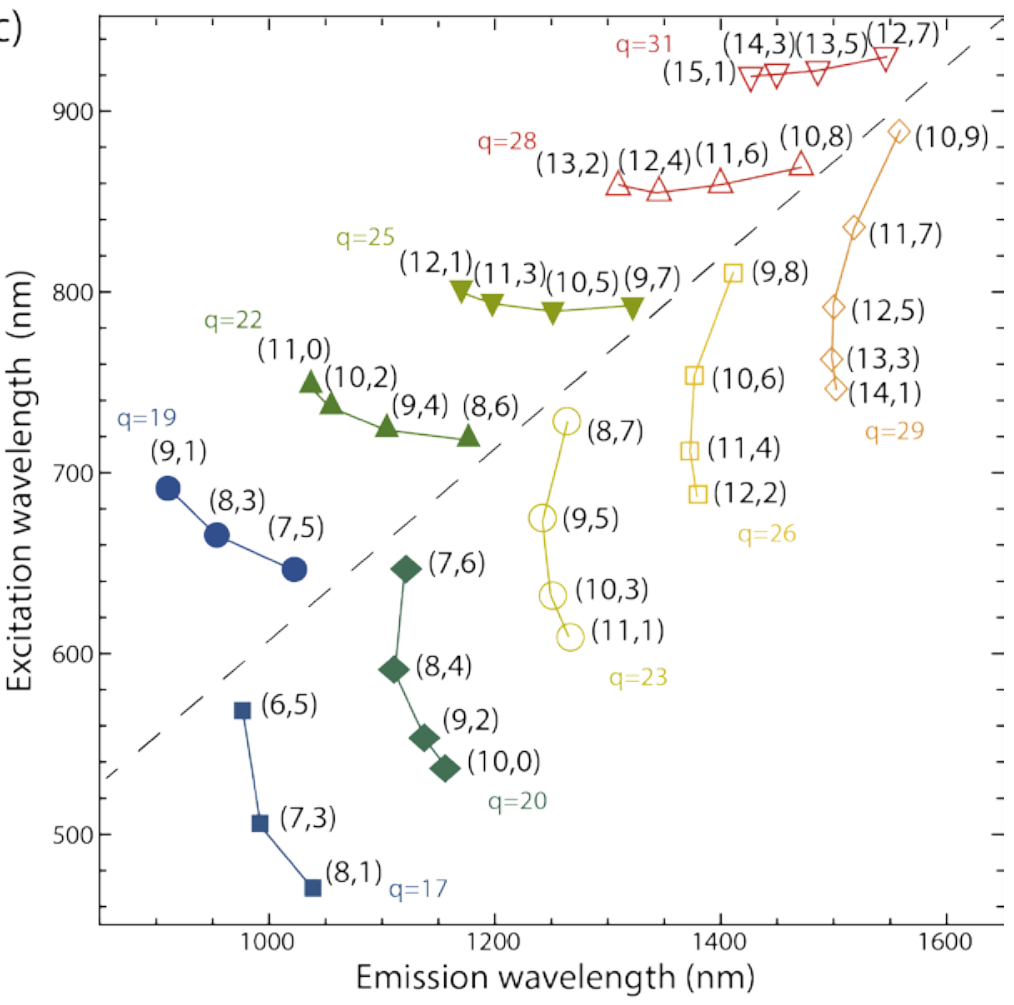

Figure 2: a) Resonant excitation of a semiconducting SWNT at the energy $E_{22}$ results, a fast nonradiative recombination towards the bottom of the conduction and top of the valence bands, into the emission of a photon with energy $E_{11}$. b) PLE chart of the $(8,4)$ SWNT species. c) Pseudo-PLE chart of the most common semiconducting SWNTs [27], highlighting the several laola families arising on the different branches in the Kataura plot.

It is worth pointing out that, even if the single-particle picture is very effective in depicting the optical process, the optical response in nanotubes is ruled out by excitons [36],[37]. Contrarily to bulk semiconducting materials, where excitonic effects demand cryogenic working conditions to be observed, the suppressed screening in such one-dimensional structures ensures excitonic binding energies that easily exceed the hundreds of $\mathrm{meV}$ and can go up the $\mathrm{eV}$ range [37],[38]. While such excitonic effects shift the expected values of the $E_{i i}$ transition energies [39], they do not change the overall morphology of PLE pattern.

As mentioned above, PLE is a powerful technique to straightforwardly assess the efficacy of a functionalization process onto the nanotube. Their one-dimensionality makes them extremely sensitive to the environment. For example, if semiconducting SWNTs are not isolated but in bundles, nonradiative processes due to tube-tube excitation transfer mechanisms will quench the tubes' emission, making possible to experimentally characterize them only through absorption or Raman spectroscopy [18]. On the other side, any conversion of the carbon atoms of the tubes' backbone from the $\mathrm{sp}^{2}$ to the $\mathrm{sp}^{3}$ hybridization state acts as localization center for the excitons and quenches their radiative emission [40]. Thus, the experimental observation of an emission spot in a PLE chart certifies that the analyzed species is as pristine as it can be, it is isolated, unperturbed, and with low defectivity. 
a)

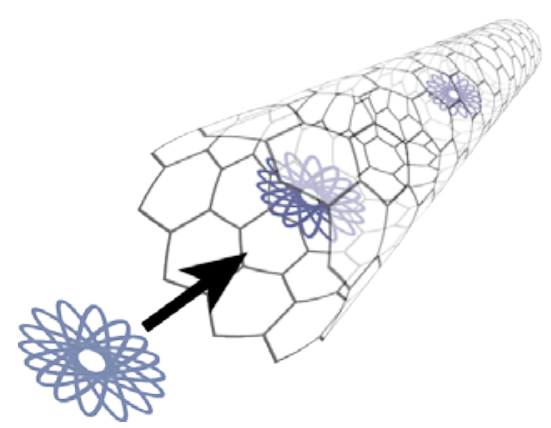

b)

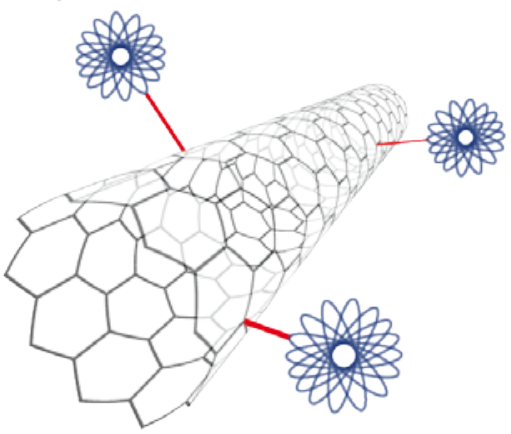

c)

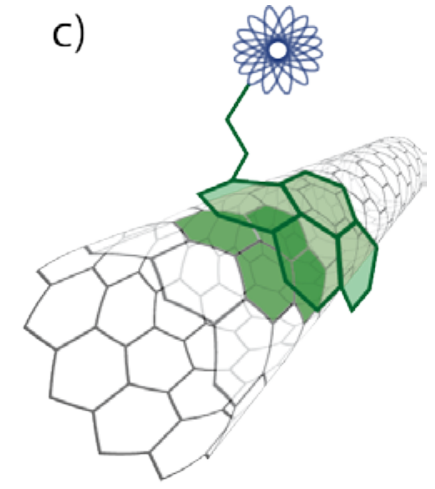

Figure 3: Schematic depiction of the a) the endohedral functionalization and $\mathbf{b}$ ) covalent and $\mathbf{c}$ ) noncovalent exohedral functionalization approaches.

\section{Functionalization strategies}

Due to their extended $\pi$-conjugated network, as-produced SWNTs form bundles hold together by $\pi-\pi$ stacking interactions. Any optoelectronic use of SWNTs would require them to be isolated and processed. It is additionally mandatory for the process to be as unperturbing as possible, in order to avoid triggering loss of coherence of the excitonic wavefunctions and emission quenching. Several functionalization strategies have been developed so far. For an exhaustive review on the topic, please refer to [21],[41]. Here we will highlight some of the most common approaches, focusing our attention on the outcome on the optical features of the SWNTs. Please note that we will use in here the term functionalization with a broader meaning, extending the definition from attaching a functional group to the tubes to include any general action performed onto the tubes with the aim to tailor them for applications. This includes, for example, SWNTs isolation, solubilization, suspension, sorting, and so on.

In Fig. 3 are summarized the principal approaches adopted to functionalize SWNTs. The very first discriminating factor is where the functionalization takes place. The endohedral functionalization aims for the filling of the inner part of the tubes, as sketched in Fig. 3a. The exohedral functionalization, on the contrary, strives for adding the functionalities onto the outer wall of the tubes (Fig. 3b,c).

\subsection{Endohedral functionalization}

SWNTs water-filling can be outlined as the most common endohedral functionalization process. It occurs during the surfactant-aided solubilization of SWNTs in aqueous environments. Due to the strong sonication powers involved in the process, the SWNT caps break and water molecules penetrate the tubes inner cavity. It is a nonperturbing process, as it does not quench the emission of the tubes, but it does anyway alter their optical response. PLE is a suitable investigation tool to distinguish 
between empty and water-filled nanotubes by monitoring the excitation and emission red-shifts caused by the presence of water [42].

The inner environment of the tubes can be exploited for several purposes. It can serve as a template for the aggregation and organization of molecules [43],[44] to yield for example giant Raman scattering response [45] or macroscopic dipole moments [46]. Due to the SWNTs stability towards thermal decomposition, the inner cavity of the tubes has even been exploited as nano-reactor environments for production of simple molecules [47] as well as more elaborate structures, such as graphene nanoribbons [48]. Despite the many advantages, the endohedral functionalization is limited by the size of the functionalities that fit into the tubes' inner diameter, which for the largest tubes used for optical experiments reaches $2 \mathrm{~nm}$.

Exohedral functionalization does not suffer of such limitation and can virtually attach functional groups of any size. The exohedral approaches can be splitted into two main classes, the covalent (sketched in Fig. 3b) and the non-covalent ones (Fig. 3c), depending upon the nature of the bond used to immobilize the functionalities onto the SWNTs sidewalls. Both approaches offer an entangled set of advantages and disadvantages.

\subsection{Exohedral covalent functionalization}

The covalent approach relies on covalent chemistry to attach the desired groups onto the tubes. While this certainly ensures strength and stability of the attachment, it does come at the cost of the structural, electronic, and optical integrity of the SWNTs. As a result of the chemical treatment, the carbon atoms on which the functional groups are attached are converted from $\mathrm{sp}^{2}$ to $\mathrm{sp}^{3}$, degrading the SWNT conjugation. This can be estimated by measuring the ratio between the D and G band in the Raman spectra of the tubes [49],[50]. Several techniques have been developed to efficient perform covalent functionalization of SWNTs [51]-[58], some of them even implementing selectivity towards

a)
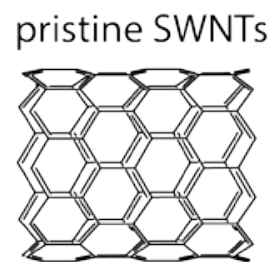

b) carboxylated SWNTs

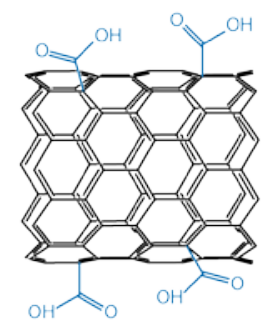

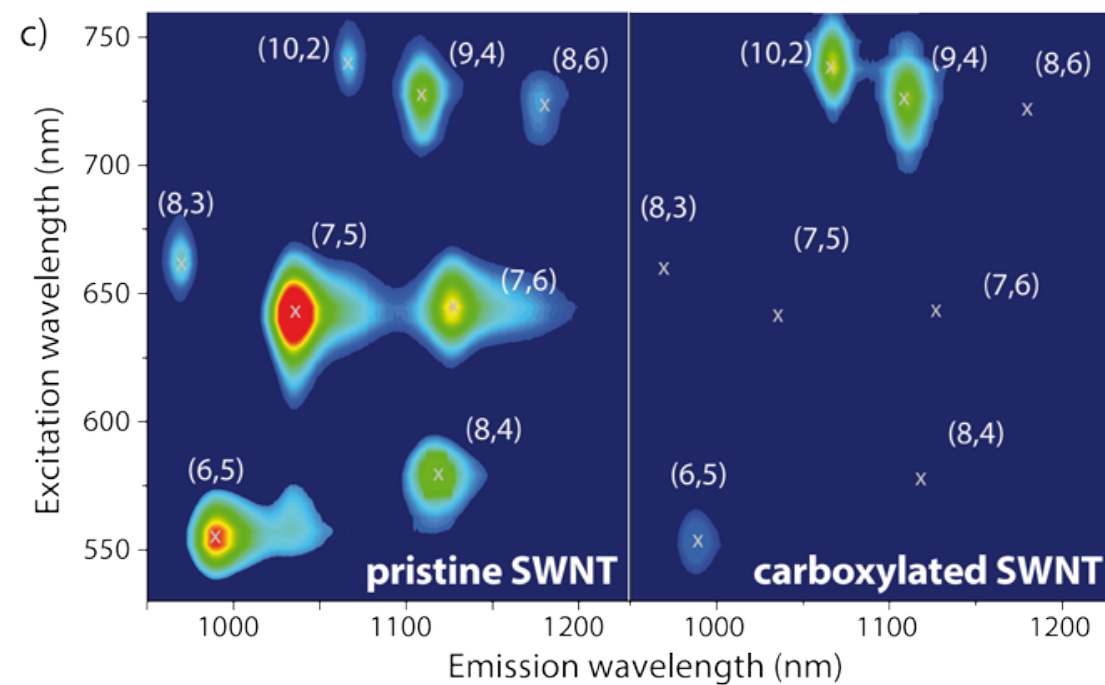

Emission wavelength $(\mathrm{nm})$

Figure 4: Molecular sketch of pristine a) and carboxylated b) SWNTs. c) Comparison of the PLE chart of pristine tubes (left panel) with tubes covalently functionalized according to protocol of Ref. [66] (right panel). 
the diameter or electronic character of the tubes [59]-[64]. A thorough review on the effects of covalent functionalization approaches, together with the discussion on the hybridization states of the derivatized carbon atomes can be found in Ref. [65]. Even mild covalent synthetic reaction conditions performed for very short times irreversibly destroy the emissive properties of the SWNTs [66]. Fig. 4 compares the emission of pristine nanotubes with the nanotubes prepared according the procedure of Ref. [66]: No emission can be observed from the majority of the tubes. This is the reason why it is dogmatically assumed by the nanotube community that covalent chemistry destroys the optoelectronic properties of SWNTs. A noteworthy exception is the aryl functionalization, in which usually inactive excitonic states, called dark excitons, are activated by the $\mathrm{sp}^{2}$ to $\mathrm{sp}^{3}$ conversion and exhibit high emission yields [67]. This has triggered new research on attaching various oxygen or aryl derivatives onto the SWNT sidewalls to activate their dark excitonic modes [68],[69]. Please note, anyway, that this comes at the cost of the structural integrity of the SWNTs, as indicated by the increase of the D/G ratio in the Raman spectra of the functionalized tubes [67]. Aryl-functionalized SWNTs are indeed mentioned as systems of reduced dimensionality that have passed from 1-d to $0-d$. Another promising covalent approach involves the SWNTs functionalization with transition metals through hexahapto bonds formation. The approach is covalent while, on the same time, preserves the $\mathrm{sp}^{2}$ character of the SWNTs [70],[71]. The effect of such functionalization on the photoluminescence emission has yet to be reported.

In general, the harsh synthetic conditions required to perform covalent chemistry cause significant increase of the SWNT defectivity. In Section 4 we will report on a novel covalent approach that does not require the conversion of the $\mathrm{sp}^{2}$ carbon atoms into their $\mathrm{sp}^{3}$ form to attach the functionality and it does not increase their defectivity while fully preserving the emissive properties of the SWNTs.

\subsection{Exohedral non-covalent functionalization}

The non-covalent approach, contrarily to the covalent one, is based on milder physisorption interactions, such as the $\pi-\pi$ stacking interactions [72],[73], polymer wrapping mechanisms [74], or micellar forces [75]-[77]. It does not perturb the $\mathrm{sp}^{2}$ network neither its $\pi$-conjugation, safeguarding the emission from the tubes. The drawback of such scheme is its instability: Changes in the environmental conditions can easily reverse the functionalization, triggering loss of the functionality and ultimately waist of the tubes by re-bundling and precipitation.

The most commonly used noncovalent treatment is the individualization and suspension of SWNTs by surfactants. Surfactants are amphiphilic molecules with hydrophilic heads and hydrophobic tails. When they exceed a certain concentration, called critical micellar concentration, they assemble together to build up complex structure called micelles with hydrophilic outer shells and hydrophobic inner cores. SWNTs are hydrophobic and can be stabilized within the micelles inner cores. There are plenty of different surfactants with heads and tails of various nature. A useful 
comparative study on the SWNTs isolating performances of different surfactants can be found in Ref. [78]. Variation of the structure of the surfactant can induce selectivity in the interaction with the solubilized tubes [79]-[88]. Selective solubilization of the SWNTs is possible through the choice of specific DNA fragments [89], polymers [90], and copolymers [91]. Another common non-covalent functionalization approach exploits small aromatic fragments, for example pyrene (sketched in green in Fig. 3c), perylene, porphyrine, and coronene. Those are some of the most common but not the only ones that adhere through $\pi-\pi$ stacking interactions onto the nanotube sidewalls. Those small fragments can serve as functionality themselves or be the anchor part of more complex moieties bearing additional functional groups to be immobilized in the proximity of the tubes.

In the coming section we will provide few examples out of the broad basin of non-covalent functionalization schemes for different applications.

\section{Examples of advanced functionalities}

\subsection{SWNTs as a biosensing platforms}

To exploit the SWNT as sensors, it is fundamental to develop an immobilization protocol that does not perturb their properties neither affects their sensitivity to the environmental changes. Chen et al., with the aim of immobilizing proteins onto the tubes, faced this challenge following the noncovalent approach by employing 1-Pyrenebutanoic Acid, Succinimidyl Ester: The pyrene part hold onto the tubes through $\pi$ - $\pi$ stacking without damaging their structure while the succinimidyl ester reacted with the amine groups of a protein to efficiently immobilize it onto the tubes [92]. To cite another example, polymer-wrapping of SWNTs not only preserves their emission but it also enhances their sensitivity. In this way, changes of the intensity of the tubes' infrared emission can be exploited for monitoring in real-time the dopamine release [93]. There is a huge literature on different applications of SWNTs for biosensing purposes. An exhaustive overview on the topic can be found in Ref. [94].

\subsection{SWNT Sorting}

Another application field in which the non-perturbing nature of non-covalent approaches is required is the sorting of SWNTs, which is compulsory for any technological application. While single-chirality controlled growth of SWNTs has been realized only for special chiralities [95], commercially available SWNTs are still chirally polydispersed within the as-produced bundles. Different appraoches such as density-gradient ultracentrifugation [96], gel dielectrophoresis [97] or the overloading technique [98] and others [99],[100] have been developed to isolate single SWNTs species according to diameter, chirality, and even enantiomeric form. Such techniques, anyway, feature very low yields despite the big material costs and time consumptions. A one-pot separation technique providing spontaneous partition of the tubes according their electronic character or diameter 
has been recently developed [101],[102]. The technique relies on the relative affinity of nanotubes with different character towards different molecules that build up immiscible aqueous phases and can be easily upscaled for massive production. For an exhaustive overview over the advancements in SWNT sorting, please refer to [103].

\subsection{Energy transfer}

An advanced functionality established onto the SWNTs is the excitation transfer from molecules adsorbed onto the tubes' sidewalls or immobilized into their close proximity. Noncovalent methods are here mandatory to avoid perturbing the optical response of the SWNTs and quenching the emission from the hybrid compounds. Backes et al. have shown that small aromatic fragments such as pyrene or perylene can successfully accomplish such tasks [104]-[114]. Ernst et al. have moreover demonstrated that, while the direct optical excitation process is chirality-selective, requiring the exciting photons to be resonating with the $\mathrm{E}_{22}$ transition of a specific tube, the adsorbate-mediated excitation of the SWNTs is not [115]-[117]. Perylene-mediated excitation transfer ensures emission from all the SWNTs the molecule is physisorbed onto, overruling the chiral selectivity of the direct optical excitation process [115]-[117]. Roquelet et al. have proven that such process occurs for porphyrin attached onto the SWNTs as well, with efficiency close to 100\% [118]-[122]. While Backes et al. and Ernst et al. exploited $\pi-\pi$ stacking interactions to immobilize their compounds onto the SWNTs, it is worth pointing out that the porphyrin immobilization pursued by Roquelet et al. has been achieved following another non-covalent method, namely the so-called micelle swelling technique. The idea behind this technique is that, if some organic solvent is added to an aqueous suspension of surfactant-stabilized SWNTs, the solvent will penetrate the micelles, swelling them. After some time, the solvent will evaporate, the micelle will shrink, and an overall improvement of the organization of the micellar structure can be observed [123]. The protocol developed by Roquelet et al. exploits the organic solvent as a vector for non-water soluble molecules, carrying them within the micelles so that, at the end of the process, they are confined within the very same micellar cores that already contain the SWNTs [118]. This technique has been shown to be very powerful for implementing advanced functionalities with molecular moieties that otherwise would not withhold in the proximity of the SWNT sidewalls on their own. One example worth mentioning is the use of micelles as nano-reactors to control and trigger polymerization reactions directly onto the SWNT sidewalls [124]. Recently, Delport et al. developed the micelles fusion as a faster and with higher yield alternative to the swelling technique to encapsulate porphyrin molecules within the SWNTs-containing micelles [125],[126].

\section{Beyond the covalent-noncovalent dichotomy}

As mentioned in Section 3, when choosing the functionalization approach to pursue, it is common expectation to face the dichotomy between the strength of the functionalization (ensured by the covalent approach) and preservation of the quantum features by the tubes (ensured in the noncovalent 
a)

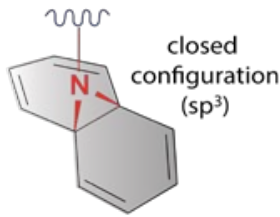

b)

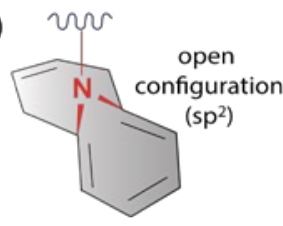

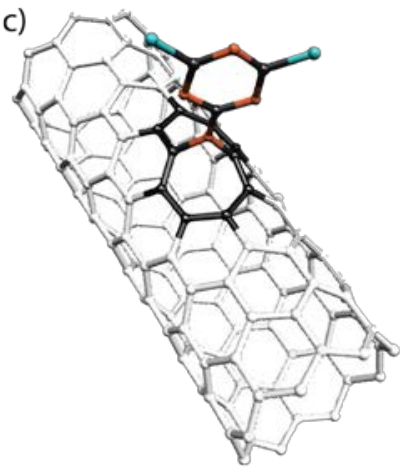

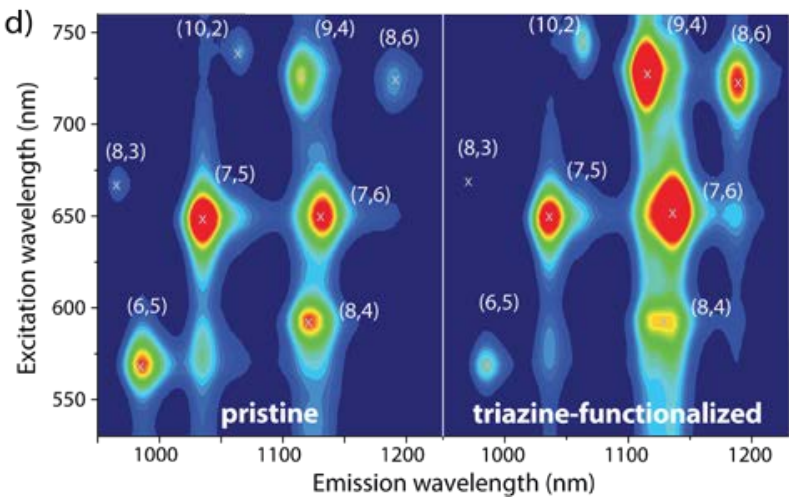

Figure 5: As a product of a [2+1] cycloaddition reaction bridging a triazine molecule, the two carbon atoms underlying the bridge can be in a) their $\mathrm{sp}^{3}$ state in the closed configuration or $\mathbf{b}$ ) their $\mathrm{sp}^{2}$ state in the open configuration. c) Quantum chemical calculations show that the open configuration is the stable one. d) Comparison between the emission of pristine (left panel) and triazine-functionalized (right panel) nanotubes. Adapted from [127].

framework). This is due to the fact that a covalent reaction results in defects, dangling bonds, and the conversion of a fraction of the carbon atoms from the $\mathrm{sp}^{2}$ to the $\mathrm{sp}^{3}$ hybridization state. This inexorably results in quenching of the tubes' emission.

To overcome such limitations, cycloaddition reactions stand up as valid candidates [127]. They do not require breaking of the $\mathrm{sp}^{2}$ network, as they are established through $\pi$ rather than $\sigma$ bonds. For a comprehensive review of the different possible cycloaddition reactions on carbon nanotubes, please refer to [128]. Please note that, anyway, the simple fact that the reaction does occur among $\pi$-electrons does not automatically ensure that the carbon atoms involved in the reaction will preserve their $\mathrm{sp}^{2}$ state in the final product and that thus the reaction would be beneficial for safeguarding the SWNTs properties. This topic has been intensively investigated theoretically [129], in particular Marzari compared the effects of the [2+1] cycloaddition of different molecular systems onto to the tubes with the aim of preserving their transport capabilities [130]-[132]. Such investigations verified that, after a $[2+1]$ cycloaddition reaction has taken place, there is a configuration in which the two carbon atoms bridging to the functionality are in the $\mathrm{sp}^{3}$ hybridization state, sketched in Fig. $5 \mathrm{a}$ and referred as closed configuration. Anyway, there is also the so-called open configuration, favored by the tubes' curvature, in which the carbon-carbon bond below the bridge is released, see Fig. 5b. In this latter configuration, the two carbon atoms are again in their $\mathrm{sp}^{2}$ state and the $\pi$-conjugation of the carbon network is preserved.

By making use of an electron-poor nitrene derivative based on an azido-dichloro derivative, a [2+1] cycloaddition reaction scheme has been developed to covalently attach the triazine-derivative onto SWNTs [127]. The reaction to bridge the triazine onto SWNTs proceeds without an activation barrier and yields the open configuration (Fig. 5c). The bridge to the functionality does not perturb the delocalization of the $\pi$-electrons over the SWNTs while making the triazine an integrated part of the 
extended conjugated network of the tubes. The reaction, moreover, does not increase the defectivity of the structure, keeping the $\mathrm{D} / \mathrm{G}$ ratio in the Raman spectra unaltered even for the highest degrees of functionalization (one functional group every 25 carbon atoms). Most importantly, the emissive behavior of triazine-functionalized SWNTs remains unperturbed by the functionalization even at the highest degrees of functionalization (Fig. 5d). These results will hopefully activate a paradigmatic change towards the nanotube functionalization, triggering the search for alternative schemes ensuring the strength of covalent bonds while preserving the $\pi$-conjugated nature of the SWNTs.

In the coming sections, we will show how to exploit the triazine-conjugated SWNTs as building blocks for the realization of novel classes of SWNTs-based hybrids by replacing their chlorine atoms with advanced functionalities.

\section{Molecular switches functionalization}

Molecular switches are molecular systems that possess two or more metastable states, each characterized by its specific chemo-physical features. The switches evolve between the different configurations against the application of external stimuli of different nature, such as temperature, light,

a)

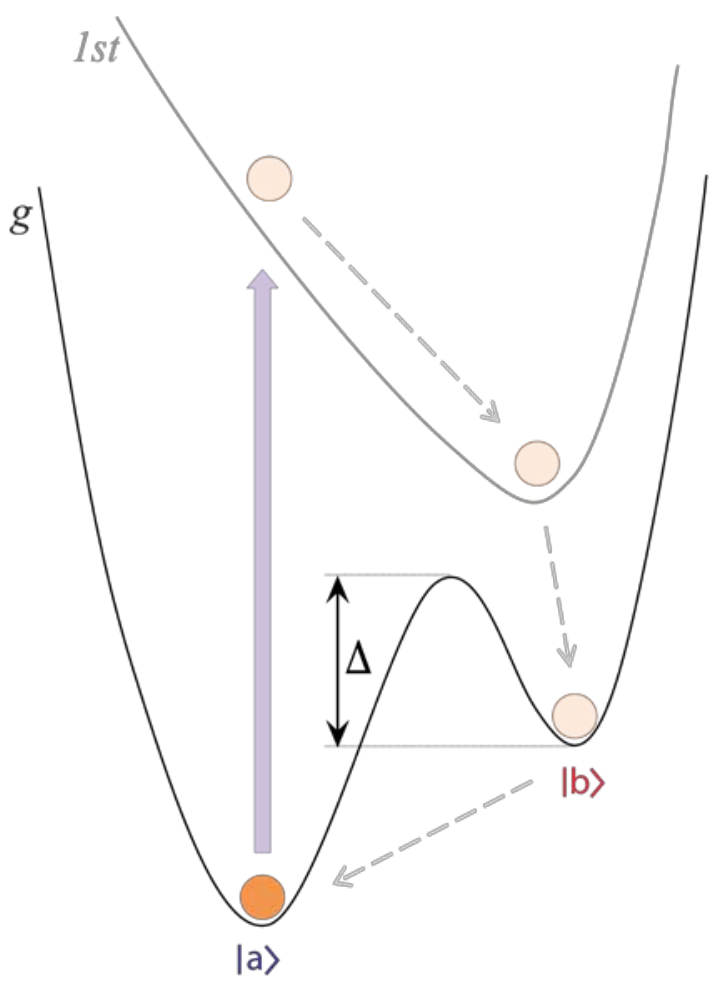

b)

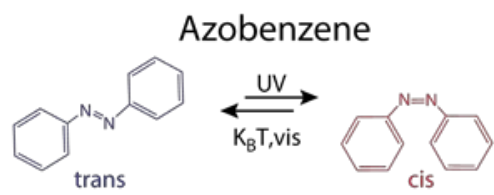

c) Spiropyran

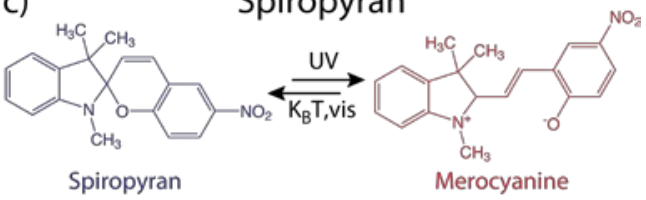

d) Dihydroazulene

e)
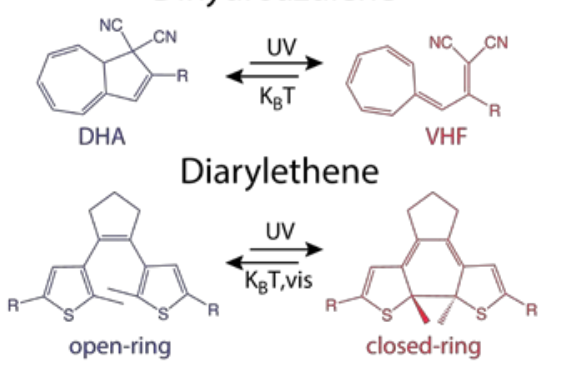

f)

Hindered alkene

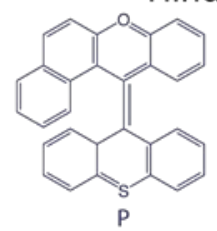

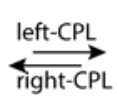

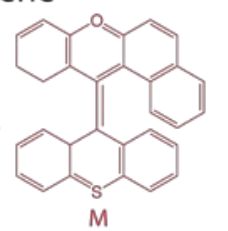

Figure 6: a) Schematic representation of the isomerization process for photochromic molecular systems. Molecular structures of some molecular switches in their most common configurations: b) Azobenzene, c) Spiropyran, d) Dihydroazulene, e) Diarylethene, and f) Sterically hindered alkenes. 
acidity, and so on. Photochromic molecular switches belong to the class of switches that react to exposure of radiation of the proper wavelength. The isomerization process occurs if the energetic landscape of their ground state possess one global minimum (corresponding to the configuration state $\mid \mathrm{a}>$ ) and one or more additional local minima ( $\mid \mathrm{b}>$, $\mid \mathrm{c}>$, and so on), corresponding to different configurations of the molecule, whereas the first excited state has only one minimum corresponding to a configuration different from $\mid \mathrm{a}>$.

The isomerization process is sketched in Fig. 6a: By shining radiation (typically photons in the ultraviolet spectral range, energetic enough to trigger molecular conformational changes) onto the system at rest in its stable configuration $\mid a>$, the molecule will lift up into its first excited state. After a fast thermalization process to the minimum of the first excited state (which is not in correspondence of $\mid a>)$, the system will decay back into the ground state in the metastable configuration $\mid b>$, from which will need to trespass the potential barrier $\Delta$ either thermally or with less energetic photons (typically in the visible spectral range) to get back to the global minimum $\mid a>$. There is a manifold phenomenology associated with the conformational change from $\mid \mathrm{a}>$ to $\mid \mathrm{b}>$.

To cite some examples, azobenzene can undergo a change of its conformation, passing from its trans to its cis form (Fig. 6b). The conformational changes between the two forms of spiropyran and dihydroazulene (Fig. 6c,d) bear big changes in intensity and direction of the molecular dipole moments. For this reason they are often referred as dipole switches. Diarylethene, on the other side, will undergo a change of its conjugation state, when passing from the ring-open to the ring-closed form (Fig. 6e), while sterically hindered alkenes will invert their chirality when exposed with circularly polarized radiation whit the proper handedness (Fig. 6f). For any further details on molecular switches, please refer to Refs. [133]-[136].

Molecular switches have been successfully exploited as building elements for photochromic lenses, sensors, and memory devices [137]-[139]. They have also been exploited as trigger elements to control the emission from fluorophores to achieve super-resolution imaging [140].

The advantage of a synthetic strategy encompassing photochromic molecular switches functionalization of SWNTs is the post-functionalization ability to affect the tubes' properties in the most not-perturbing way: Just by exposing the samples to the radiation of the proper wavelength, the switches will undergo their conformational change, displaying different chemo-physical features and altering their influence on the tubes. For example, if the isolation and solubilisation of the SWNTs are performed through surfactants comprising switching elements, it is possible to trigger re-bundling and precipitations of the tubes just by exposure to ultraviolet light. This has been proven to occur irreversibly for a stilbene-derivative [141] and reversibly with an azobenzene-derivative [142]. Please note that to ensure the isomerizing ability of the surfactants, it is important to engineer them avoiding any energetic overlap between the switch and the other parts of the compound. As a negative example, 
in a surfactant bearing a pyrene anchor, an azobenzene core, and a polyglycerol dendritic head, the energetic bands of the pyrene anchor and the azobenzene core overlapped. As a consequence, instead of getting de-excited through the relatively long-lived conformational change process, the molecule underwent fast de-excitation through radiative emission from pyrene, losing the azobenzene isomerization ability [143]. The transport properties of devices made out of azobenzene-coated SWNTs have moreover been successfully exploited for implementing color-detecting nanodevices [144].

Another switch functionalization concept aiming at influencing in a controlled and reversible way the optoelectronic properties of the tubes involves the action of dipole switches. As a matter of fact, the dipole moment of the switches couple with the nanotube carriers via Coulombic interactions. Changes in direction and intensity of the dipolar fields after isomerization are thus expected to influence the response of the tubes. There are few works in literature reporting about the interaction of SWNTs with derivatives of the dyhydroazulene (DHA/VHF) dipole switch [145],[146], whereas the functionalization with the spiropyran dipole switch has been extensive object of investigation in literature. For this reason we will discuss it in more detail in the next section.

\subsection{Spiropyran-based functionalization}

Spiropyran (SP) owns its name to the $\mathrm{sp}^{3}$ spiro-carbon atoms that connects the two halves of the molecule, the benzopyran and the indole, which result oriented almost orthogonally with respect to each other (Fig. 7a). This makes spiropyran not able to directly physisorb onto the SWNTs sidewalls on its own, as flat aromatic fragments would do. For this reason, any SPbased functionalization requires additional specific synthetic strategies to bind SP onto the SWNTs. Exposure to UV radiation optically cleaves the bond between the oxygen and the spiro-carbon, which converts to the $\mathrm{sp}^{2}$ hybridization state. The resulting structure, merocyanine (MC), is a flat molecule with the benzopyran and the indole halves oriented coplanarly. The $\pi$-electron released upon isomerization is confined within MC and gives rise to the characteristic visible absorption band of merocyanine peaked at ca. $590 \mathrm{~nm}$ (Fig. 7b).

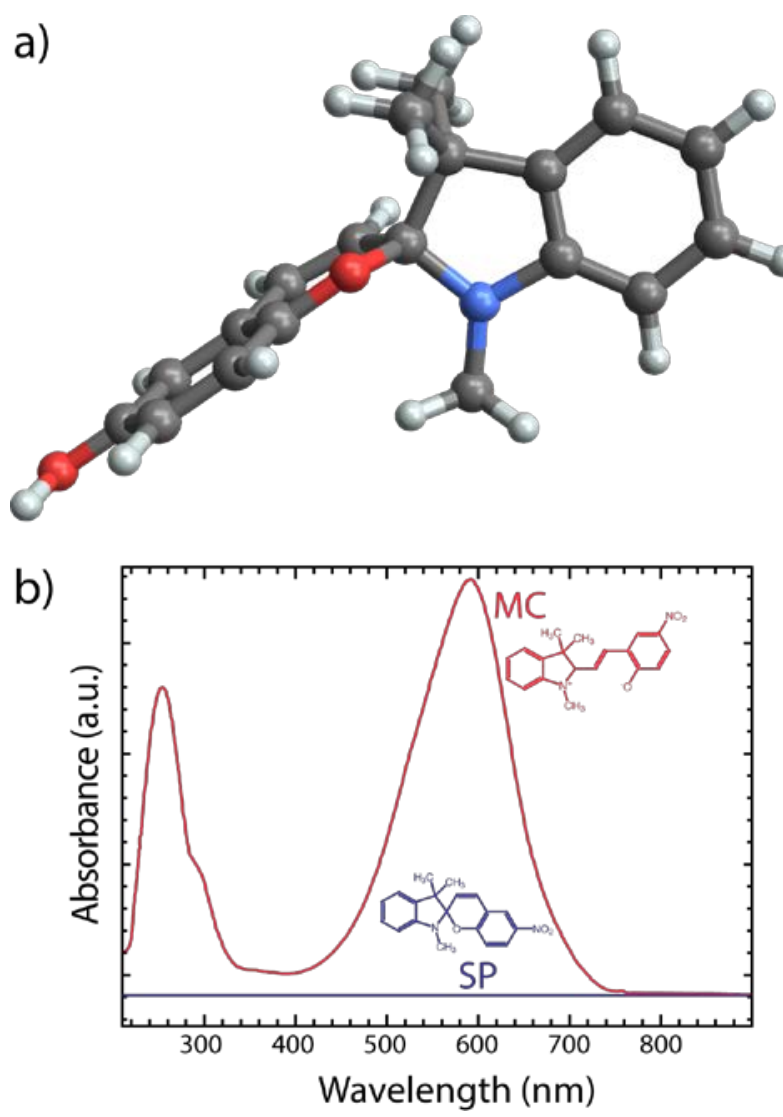

Figure 7: a) Three-dimensional molecular structure of spiropyran. b) Comparison between the absorbance of spiropyran (blue curve, labelled as $\mathrm{SP}$ ) and merocyanine (red curve, labelled as MC). 
This band, together with the other one located in the UV, is typically exploited to monitor the isomerization state of the dipole switch. For further details on spiropyran/merocyanine, please to Ref. [147].

The first attempts to conjugate spiropyran onto the SWNTs, both covalently and noncovalently, could not provide any evidence of emission from the tubes [148]-[150]. It is worth pointing that Giordani and coworkers exploited SP covalently attached onto the SWNTs to achieve light-controlled release of zinc ions for anti-inflammatory therapeutic purposes [151]. Fig. 8a sketches the molecular structure of the compound ad-hoc synthesized to preserve the emission from the tubes and noncovalently immobilize the dipole switch onto the tubes. It comprises a pyrene anchor (highlighted in green) and a chain (black) to connect it to the dipole switch (SP form in blue and MC in red). The length of the chain sets the switch-anchor separation. The compound has been able to isolate and solubilize the SWNTs while safeguarding their emission features [152].

Malic et al. developed a microscopic model to investigate the effect of the coupling of a dipolar field onto the carriers into the SWNTs, predicting a red-shift of several meV of the tubes' excitonic features due to the conformational change of the dipole switch [153]. Among the possible SWNTsdipole orientations, the biggest shifts were predicted to occur for dipoles oriented perpendicularly to the tubes' axis and for an optimal switch coverage of $0.25 \mathrm{~nm}^{-1}$. No significant effects were to be ascribed to the dipole distribution: Switches randomly distributed, as in real-world experiment would assemble, experience the same shifts of homogeneously distributed ones. The most relevant parameter is thus the tube-switch relative distance. The separation of ca. $1 \mathrm{~nm}$ between dipole and SWNTs induced small shifts in the absorption bands of the SWNTs [154], whereas no shifts in the emission could be detected. To increase the intensity of the dipole-exciton Coulombic interaction, a compound with shorter separation (ca. $0.6 \mathrm{~nm}$ ) was synthesized [155],[156]. The stronger interaction between the dipole switch and the SWNTs stabilizes the MC form of the compound, as indicated by the increase of the MC-to-SP thermal back isomerization time and by the increased red-shift of MC visible band (cfr. Fig. $8 \mathrm{~b})$, due to the enhanced interaction between the MC $\pi$-electron and the conjugated network of the SWNTs [155],[156].

The ultimate limit of this approach is provided by the micelle-swelling technique introduced in Section 4.3, which ensures direct contiguity between the dipole switches and the SWNTs. By applying this technique, Glaeske et al. observe that SWNTs coated with SP, which is transparent and with a negligible dipole moment, exhibit no shifts of their transition features, even at high switch concentrations. The big dipole moment of MC, on the contrary, induces significant shifts that increase by increasing the tubes' coverage. Such shifts in both excitation and emission features of the tubes (Fig. 8c) [157], validating the predictions by Malic. 
a)

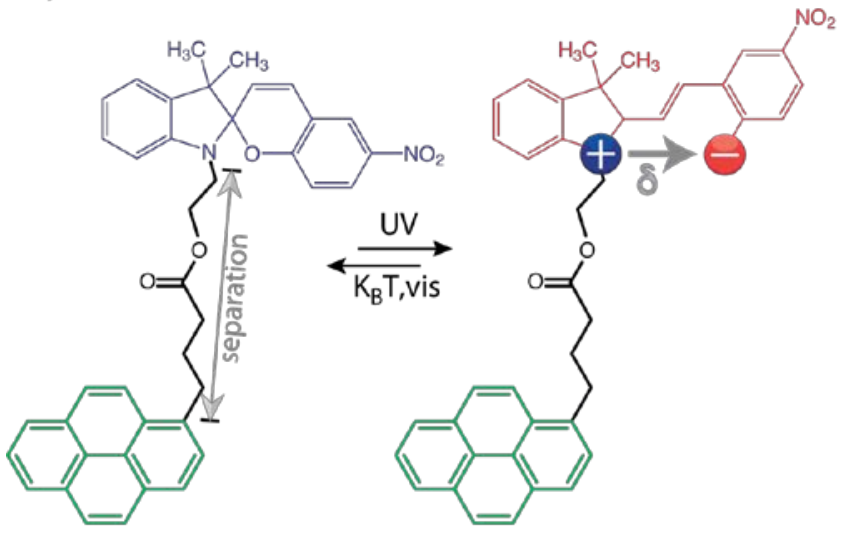

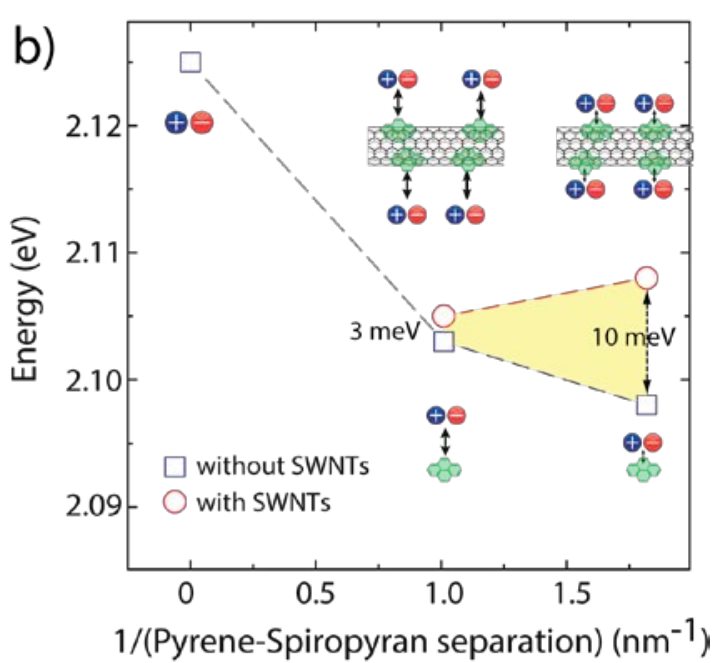

$1 /$ (Pyrene-Spiropyran separation) $\left(\mathrm{nm}^{-1}\right)$
C)

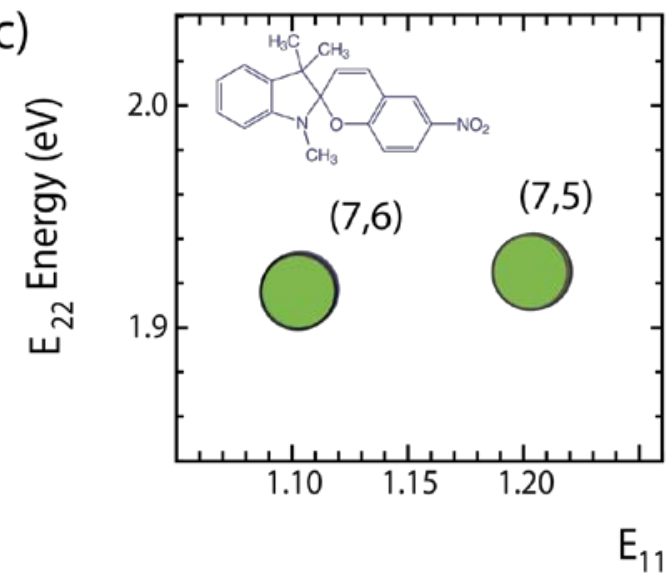

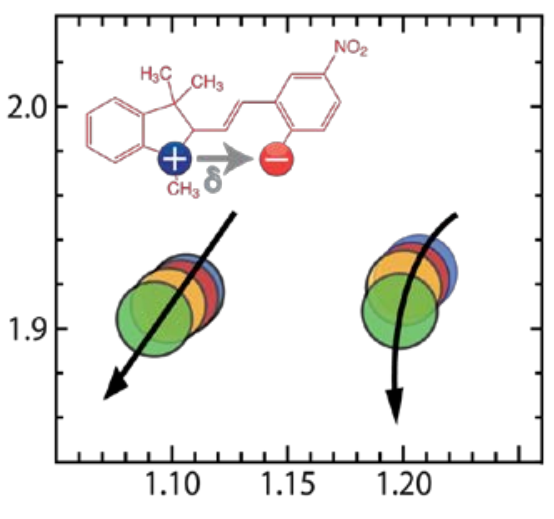

$E_{11}$ Energy (eV)

Figure 8: a) Molecular structure of the pyrene-spiropyran derivative used to solubilized SWNTs in Ref. [152]. b) Comparison of the position of the MC absorption band between the free molecule and after immobilization at different separations from the SWNTs (adapted from [156]). c) Comparison between the pseudo-PLE maps of the $(7,5)$ and $(7,6)$ SWNT species coated with spiropyran and merocyanine following the micelle-swelling technique (adapted from [157]).

These results are based on noncovalent functionalization schemes. Novel intriguing effects arise when conjugating SP onto the SWNTs covalently-functionalized with triazine introduced in Section 5 and sketched in Fig. 9a [127]. Due to the full conjugation between SWNTs and functionality, the MC's $\pi$-electron is not confined within MC anymore but can delocalize through the whole extended structure. Accordingly, the transition energy associated with its absorption band shifts towards smaller energies, out of the experimental measurement window (Fig. 9b). The uninterrupted conjugation, thus, completely changes the phenomenology of the SP-SWNTs hybrids, which become something more than the mere sum of their starting components. Being the $\pi$-electron of merocyanine not localized onto the MC structure makes it loose its strong zwitterionic character. It does not interact with the SWNTs through the dipole-dipole mechanisms anymore but rather through direct charge-carrier release. The net effect of the isomerization, indeed, is the light-triggered release of charges from the MC towards the tubes. This is confirmed by the Raman spectra, which exhibit the characteristic shifts of the G band vibration associated with SWNTs doping (Fig. 9c). The most striking effect of the 
a)

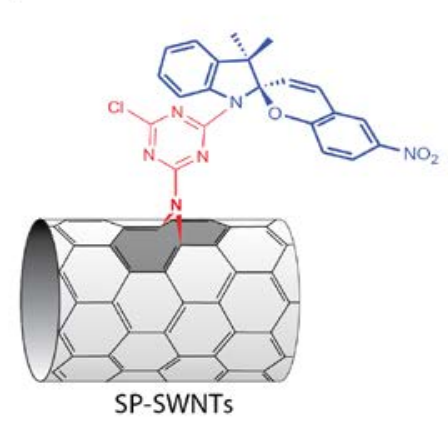

b)

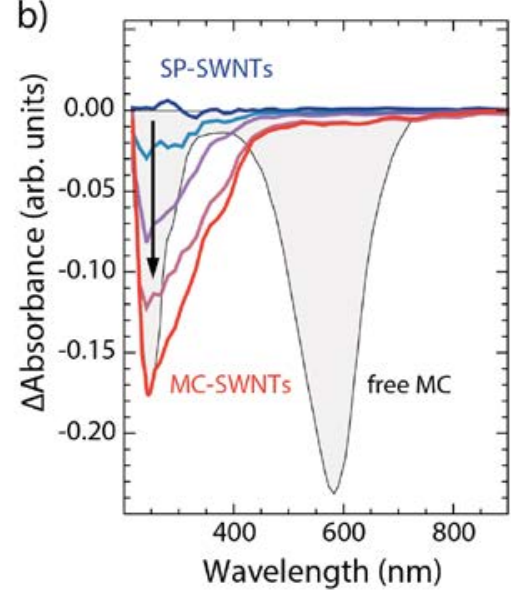

c)

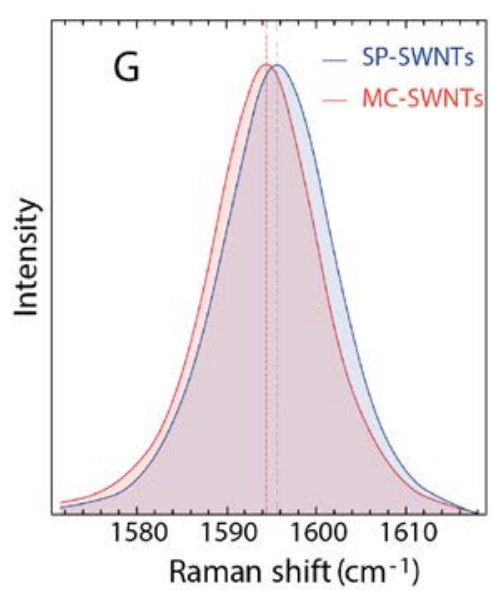

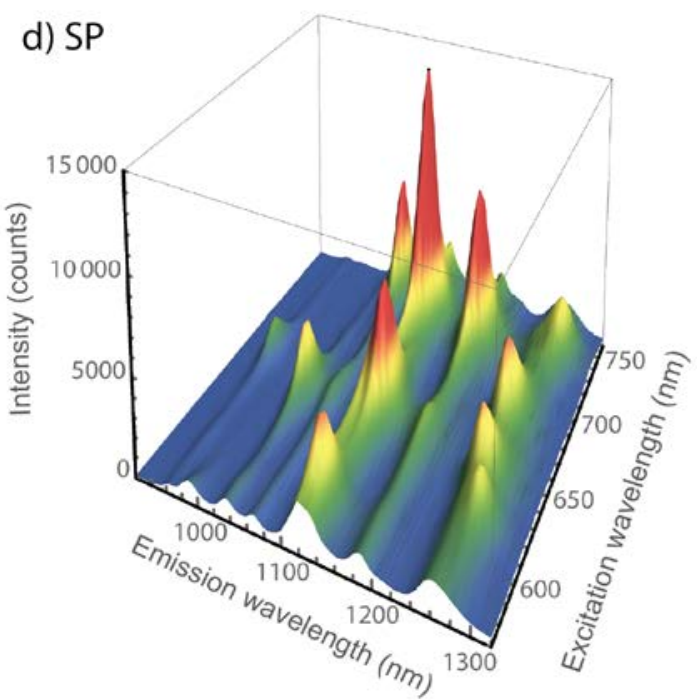

e) $M C$

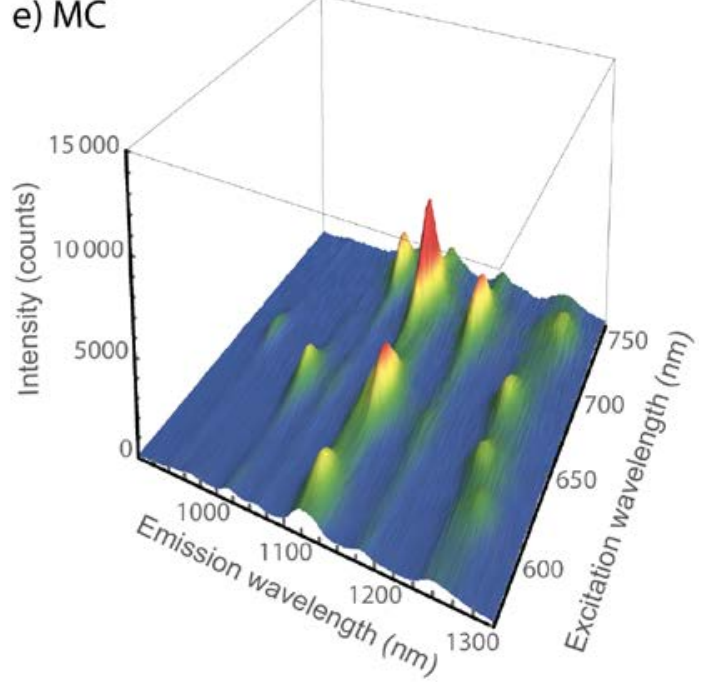

Figure 9: a) Schematic structure of the sapiropyran conjugated hybrid SP-SWNTs. b) Absorption spectrum of the SP-SWNTs under exposure to UV radiation. The grey curve is the absorption of free MC. c) Comparison between the Raman G bands of SP-SWNTs and MC-SWNTs. Comparison between the PLE maps of SP-SWNTs (d) and MC-SWNTs (e). Pictures taken from [127].

release of free charges within the tubes, anyway, is the increase of the probability of non-radiative recombination events resulting in a net quenching of the emission from the SWNTs (Fig. 9d,e). For more details, please refer to [127]. The light-triggered control of the SWNTs' charge carrier in density opens up new perspectives for advanced optoelectronic applications.

This example just highlights one of the many possible applications of SWNTs functionalized with molecular switches to affect in a controlled and reversible way their properties. In the next section we will describe another advanced functionalization scheme of the SWNTs developed to enhance their optical response by making use of plasmonic nanosystems.

\section{Nanoplasmonic hybridization}

Despite their outstanding capabilities, the actual employment of SWNTs for optoelectronic and biological applications is frustrated by the tubes' low emission yields, generally below $1 \%$ and above 
only in very exceptional contingencies [158]-[160]. The origin of such a low yield resides in the fact that the lowest-energy exciton in SWNTs is nonradiative [161]. While the aryl functionalization described in Section 3.2 efficiently activates such dark excitonic state, it still degrades the crystalline structure of the tubes. To escape this boundary, SWNTs have been embedded in special optical environments such as cavities [162],[163] or photonic crystals [164] to exploit their ad-hoc designed electromagnetic modes. Another suitable approach comprises plasmons, the special electromagnetic modes arising from the collective free-electrons oscillations in metals and localized close to the metal surface. Plasmons have the ability to concentrate electromagnetic radiations over regions of space much smaller than the ones obtained by traditional optics, going beyond the diffraction limit. The fields are not only confined in space, they get also enhanced in intensity. In the proximity of the socalled plasmonic hot-spots, the local intensity of the radiation can increase by order of magnitudes with respect to the pump intensity. For a thorough overview on plasmonics, please refer to [165]. Heeg et al. for example exploited the enhanced cavity mode of a gold dimer to enhance the Raman scattering of SWNTs placed within the cavity [166]. Enhancement of the radiative emission from SWNTs has been moreover achieved by depositing SWNTs onto rough gold surfaces [167],[168]. This technique, anyway, did not offer the possibility of controlling the SWNTs allocation in proximity of the surface plasmonic hot-spots. Zaumseil and coworkers have pursued a more rational approach by depositing SWNT films onto a plasmonic cavity made out of arrays of metallic particles created by ebeam lithography, which produced evidence of cavity polaritonic coupling [169],[170].

All of the methods highlighted so far are based on top-down techniques. Alternatively, bottom-up approaches involving the wet-chemical synthesis of colloidal metallic nanoparticles have started developing for different purposes. For example, gelatin-stabilized gold nanoparticles immobilized onto SWNTs have been successfully exploited for cytosensing and drug uptake [171]. The most interesting feature of colloidal Au nanoparticles, anyway, is that their plasmonic modes do not require special excitation configurations to be activated; they simply are directly activated by exposure to standard electromagnetic radiation [172]. Moreover, the frequencies of their plasmonic modes overlap with the optical transition energies of the SWNTs. The phenomenology of the interaction between an emitter and a plasmonic particle changes when changing their relative separation. The presence of the metallic nanoparticles can activate nonradiative additional de-excitation channels.
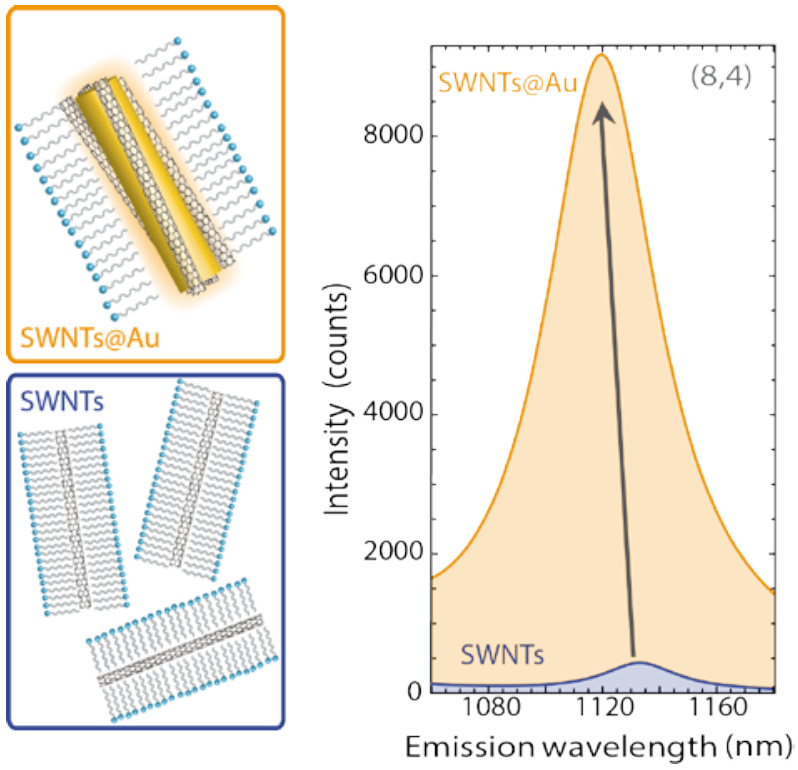

Figure 10: After hybridization with gold nanorods, the $(8,4)$ SWNT species enhances by a factor of 20 its emission intensity. Adapted from [174]. 
Moreover, the emitter can sense the localized near fields or the propagating radiation scattered from the metallic particle. At very short emitter-particle separations, the dominating component is the near field surrounding the metallic particles' surface. For bigger separations, the contribution of the propagating radiation becomes dominant and can give rise to emission quenching or enhancement, depending upon the wave-vectors-matching between the radiation generated by the emitter and the plasmonic modes [173]. The experimental configuration will set which of those mechanisms rules the emitter-plasmon interaction phenomenology. By extending the micelle-swelling technique, Glaeske et al. have encapsulated SWNTs within the same micelle already suspending Au nanrods, warranting close Au nanorods-SWNTs separations. The technique ensures homogeneous control of the exposure of any SWNT to the plasmonic near-field of the metallic particle, yielding a 20 -fold enhancement of the nanotubes emission (Fig. 10) [174],[175]. In such configuration, two mechanisms can contribute to the enhancement of the radiative emission from the tubes: Stronger pumping through the enhanced near-field of the Au plasmon or increased radiative de-excitation rate. Transient-absorption investigation revealed for those colloidal nano-plasmonic hybrids systems the enhanced absorption to be the leading mechanism for an efficient emission enhancement [176]. When placed in the very close proximity of metallic surfaces, thus, SWNTs can act as optical read-out of the plasmonic near fields.

\subsection{Bottom-up, covalent strategy}

While very profitable for its flexibility, the method based on the micelle encapsulation, as most of the non-covalent strategies, is intrinsically unstable under changes of the environmental conditions. Deposition of the samples onto a solid substrate causes for example the hybrids to be destroyed. Covalent attachment methods are thus more promising for applications requiring substrate deposition rather than suspension in solution. Attaching the plasmonic nanoparticles through a variation of the cycloaddition-based triazine conjugation introduced in Section 5 can be extremely profitable for such applications, as it would ensure the strength of the covalent bonds together with the preservation of the pristine optoelectronic properties of the tubes. Fig. 11a sketches the molecular structure of the covalently triazine-functionalized SWNTs with the chlorine atoms replaced by thiol groups to promote immobilization of the Au nanoparticles along the SWNTs sidewalls. The TEM micrograph in Fig. 11b shows the Au@SWNT hybrids obtained through the covalent attachment method; the necklace-like assembly of $\mathrm{Au}$ nanoparticles attached along the SWNTs is clearly visible [127]. The covalent Au@SWNTs hybrids are very robust towards environmental changes and do not get destroyed after deposition onto substrates. Fig. 11c compares the solution of the pure Au nanoparticles (left panel) with the Au@SWNTs hybrids (right panel), where the longitudinal coupling between the Au particles due to their assembly along the nanotube axis is responsible of the colour change of the suspension.

The proximity of the metallic nanoparticles make the SWNTs feel the enhanced near field localized around the nanoparticles' surface. As a consequence, the enhanced absorption from the 
a)

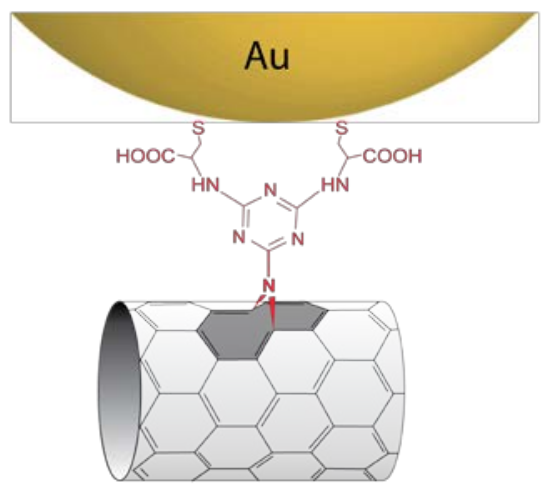

b)

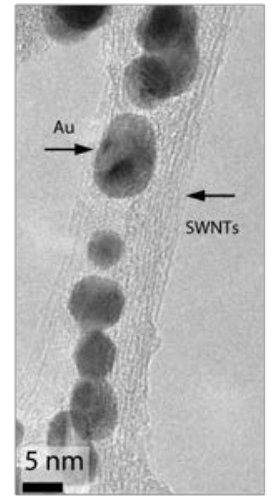

e)

d)
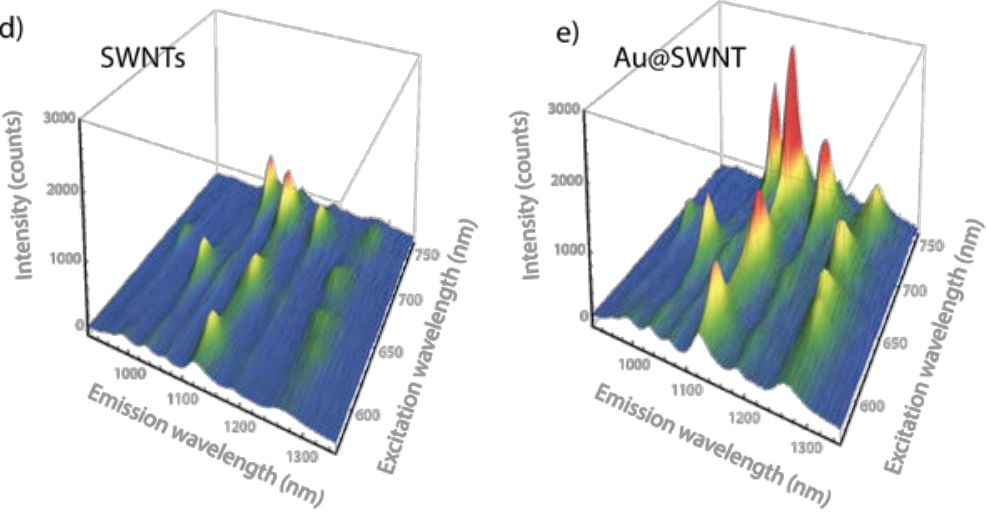

c)

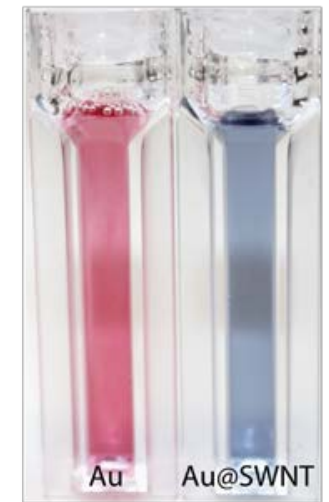

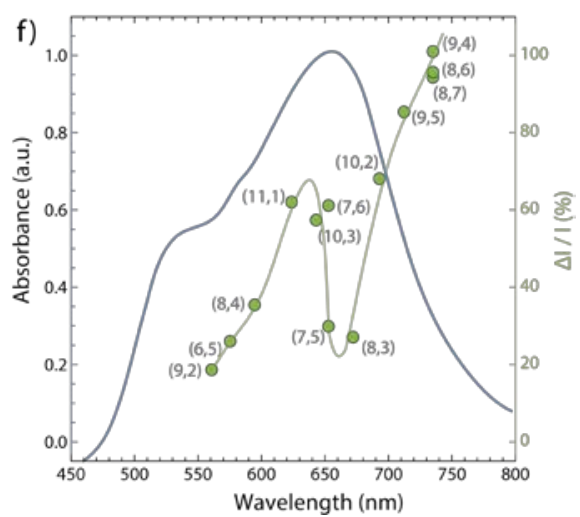

Figure 11: a) Molecular sketch of the Au nanoparticles anchored to the triazine-functionalized SWNTs. b) TEM micrograph of the Au@SWNTs hybrids. (c) Comparison between the suspension of pure Au nanoparticles and Au@SWNTs hybrid. After attachment of the Au nanoparticles onto the SWNTs, the solution changes color. PLE maps of the SWNTs before (d) and after (e) hybridization with the Au nanoparticles. (f) Comparison between the absorption spectrum (blue curve) and the relative intensity increase of various Au@SWNT hybrid species (green dots, the line is a guide to the eye). Pictures taken and adapted from [127].

plasmonic field grants enhancement of the radiative emission from the Au@SWNTs hybrids, cfr. Fig 11d,e. Please note that the $\pi$-preserving nature of the functionalization approach is a key element in these experiments. Similar results would not have been possible by pursuing standard covalent functionalization techniques to bind the metallic nanoparticles onto the SWNTs [127].

As mentioned above, SWNTs can be exploited in such systems as optical read-outs of the plasmonic near-field, converting them from localized fields into propagating radiation. It is interesting to compare the enhancement of profile of the different SWNTs species within the hybrids (Fig. 11f, green) with their absorption spectrum (Fig. 11f, blue): Both exhibit qualitatively the same trend but with a significant red-shift of the emission resonance profile with respect to the absorption spectrum. This is a clear signature of the near-field mediated character of the exciton-plasmon interaction leading to the observed enhancement behaviour [127],[176],[177], similarly to the behaviour already reported in simpler molecular systems [178]. These results encourage development of further investigation along this synthetic strategy to tailor, gain better insights, and rationalize the exciton-plasmon interaction in such hybrid nanoplasmonic systems. 


\section{Conclusion}

SWNTs are materials with outstanding mechanical and optoelectronic properties. The very same factors that make them extraordinarily promising, anyway, work also as hindrance, interfering against their employment in applications. Their conjugated $\pi$-network imbues them with exceptional optical and electronic properties but on the same time makes them strongly bundle together, blurring out most of their quantum peculiarities. A huge basin of different strategies has been developed to circumvent this problem and make nanotubes ready for applications. In this review, we concentrated our attention onto the effects of the functionalization onto the properties of the tubes, highlighting the compromises that one has to accept between strength of the functionalization and preservation of the quantum optoelectronic properties of the tubes. Among the different experimental techniques available to characterize SWNTs, we have remarked that excitation photoluminescence spectroscopy (PLE) is the most straightforward one to exclude damages due to the functionalization process. Among the different functionalization approaches, we mostly focused and compared the exohedral noncovalent $v s$. covalent functionalization approaches. While the noncovalent does not destroy the optical emission from the tubes, it is generally assumed that a dichotomy reigns between the covalent approach and emission from the tubes, with the only exception of the aryl functionalization, which activates dark excitonic modes but at cost of the conversion of the carbon atoms into their $\mathrm{sp}^{3}$ hybridization state. This assumption originates from the typically harsher conditions at which the covalent reactions take place, which favour the creation of defects, dangling bonds, etc. This anyway does not have to be necessarily the case for covalent chemistry on SWNTs. We reported here the case of a functionalization approach performed through [2+1] cycloaddition reaction, which covalently attaches a triazine-derivative onto the SWNTs while fully preserving their $\pi$-conjugation.

In the final part of this review we highlighted some examples of advanced functionalization schemes. Without pretending to provide a complete overview of all the possible functionalization applications, we briefly introduced the sorting of single nanotube species, biosensing, and energy transfer hybrids. We moreover devoted attention to two additional functionalization schemes: a) Conjugation with molecular switches and b) Integration with plasmonic nanosystems.

Molecular switches change their properties upon external stimuli. Their attachment onto SWNTs offers degrees of freedom onto the control of the properties of the tubes not achievable by standard functionalization techniques. Precipitation of SWNTs for example can be triggered by exposure to radiation of an appropriate wavelength. By exploiting the dipole switch spiropyran/merocyanine, we have moreover shown that the control can be achieved on more fundamental properties of the tubes, such as their excitonic binding energies, their emission yield, or their doping level. These results show the potentialities of the switch-coated SWNTs for advanced optoelectronic applications. 
Nanoplasmonic systems, on the other side, have the ability of manipulate the light in ways not achievable by standard optical means. By exploiting colloidal metallic nanoparticles, which localize the electromagnetic fields in nanometric regions and enhance their intensity, we have shown that is possible to achieve enhancement of the optical response of the SWNTs. As they typically suffer of very low emission quantum yields, the hybridization with plasmonic nanoparticles opens up the way for hybrids with strong infrared emission for applications in telecommunication and for bio-imaging purposes.

\section{Future outlook}

Up to now, when selecting a functionalization strategy to adopt, one had to accept a reasonable compromise between strength of the functionalization and preservation of the nanotubes' properties. Several alternative functionalization strategies are emerging to circumvent the covalent-noncovalent dichotomy in the SWNTs functionalization. They will hopefully catalyse a dogmatic change in the conception of covalent strategies onto SWNTs and trigger novel covalent approaches to customize the tubes while preserving and tailoring their optoelectronic properties. For example, the nitrene-based cycloaddition of triazine derivatives onto SWNTs has demonstrated the capability of fine-tuning of the doping level just by controlling the degree of functionalization: By increasing the number of functional groups conjugated onto the tubes, the number of nitrogen atoms that become integrating part of the $\mathrm{sp}^{2}$ carbon network increases, lifting up the SWNTs electron density and n-doping them. Developing alternative functionalization schemes including other elements such as boron within the $\mathrm{sp}^{2}$ carbon network would help achieving controlled p-doping of the SWNTs as well. Ideally, this could lead to the implementation of a single p-n junction out of a single nanotube by controlling the functionalization yield at the single particle level.

The examples of molecular switches-based in the functionalization approaches described in in this review show that many properties of the switches-coated tubes can be altered in a controlled and reversible way. The ability to change the electronic properties in ballistic conducting channels makes SWNTs valid candidates for replacing silicon in future generations electronics and optoelectronics. Moreover the ability to switch on and off their excitonic emission under the action of external stimuli makes them appealing for sensing purposes as well as for super-resolution imaging.

Hybridization of SWNTs with plasmonic nanoparticles has been shown to enhance the SWNTs' emission yield, substantially increasing the attractiveness of SWNTs for bio-imaging purposes. The ability of SWNTs to work as optical read-out of the localized plasmonic near fields, transducing the near-fields to propagating far-fields makes nanotubes promising investigation tools for the fundamental investigations of effects on local plasmonic fields. No external perturbing elements such as cantilever tips and other scattering sources would be needed for the far-field readout, making them ideal model-tools, useful in various other systems relying on such short-range interactions. An 
example are tip-enhanced experiments, in which the role of the overlap between the energetic structure of the emitter and the plasmonic field generated by the metallic cantilever tip still requires systematic investigation.

\section{Acknowledgements}

We gratefully acknowledge the German Research Foundation (DFG via SFB 658, subprojects A6) as well as by the Focus Area Nanoscale of the Freie Universität Berlin for financial support. Special thanks are directed to Prof. S. Reich, M. Glaeske, and Dr. P. Bluemmel for their precious advice and support.

\section{References}

[1] Iijima S 1991 Helical microtubules of graphitic carbon Nature 354, 56.

[2] Durkop T, Getty SA, Cobas E and Fuhrer MS 2004 Extraordinary Mobility in Semiconducting Carbon Nanotubes Nano Lett. 4, 35.

[3] Rueckes, T, Kim K, Joselevich E, Tseng GY, Cheung CL, Lieber CM 2000 Carbon nanotubebased nonvolatile random access memory for molecular computing Science 289, 5476.

[4] Shulaker MM, Hills G, Patil N, Wei H, Chen H-Y, Wong HSP and Mitra S 2013 Carbon nanotube computer Nature 501, 526.

[5] Hamo A, Benyamini A, Shapir I, Khivrich I, Waissman J, Kaasbjerg K, Oreg Y, von Oppen F and Ilani S 2016 Electron attraction mediated by Coulomb repulsion Nature 535, 395.

[6] Pugno NM 2006 On the strength of the carbon nanotube-based space elevator cable: from nanomechanics to megamechanics Journ. Phys. Cond. Matt. 18, S1971.

[7] Iwata K, Yamazaki S, Mutombo P, Hapala P, Ondráček M, Jelínek P and Sugimoto Y 2015 Chemical structure imaging of a single molecule by atomic force microscopy at room temperature Nat. Comm. 6, 7766.

[8] Wang F, Dukovic G, Brus LE and Heinz TF 2005 The optical resonances in carbon nanotubes arise from excitons Science 308, 838.

[9] Avouris P, Freitag M and Perebeinos V. 2008 Carbon-nanotube photonics and optoelectronics Nat. Photonics 2, 341.

[10] Ma X, Hartmann NF, Baldwin JKS, Doorn SK and Htoon H 2015 Room-temperature singlephoton generation from solitary dopants of carbon nanotubes Nat. Nanotechnol. 10, 671.

[11] Gelin MF; Bondarev IV and Meliksetyan AV 2014 Optically promoted bipartite atomic entanglement in hybrid metallic carbon nanotube systems Journ. Chem. Phys. 140, 064301.

[12] Sharma A, Singh V, Bougher TL and Cola BA 2015 A carbon nanotube optical rectenna. Nat. Nanotechnol. 10, 1027. 
[13] Hong G et al. 2012 Multifunctional in vivo vascular imaging using near-infrared II fluorescence Nat. Med. 18, 1841.

[14] Welsher $\mathrm{K}$ et al. 2009 A route to brightly fluorescent carbon nanotubes for near-infrared imaging in mice. Nat. Nanotechnol. 4, 773.

[15] Hilder TA and Hill JM 2008 Carbon nanotubes as drug delivery nanocapsules Curr. Appl. Phys. 8, 258.

[16] Gu YJ, Cheng JP, Jin JF, Cheng SH, Wong WT 2011 Development and evaluation of pHresponsive single-walled carbon nanotube-doxorubicin complexes in cancer cells Int. Journ. Nanomed. 6, 2889.

[17] Saito R, Dresselhaus G and Dresselhaus MS 2008 Physical Properties of Carbon Nanotubes (London: Imperial College Press).

[18] Reich S, Thomsen C and Maultzsch J 2004 Carbon Nanotubes: Basic Concepts and Physical Properties (Weinheim: Wiley).

[19] Saito R, Fujita M, Dresselhaus G and Dresselhaus MS 1992 Electronic structure of graphene tubules based on $\mathrm{C}_{60}$ Phys. Rev. B 46, 1804.

[20] Kleiner A and Eggert S 2001 Curvature, hybridization, and STM images of carbon nanotubes Phys. Rev. B 64, 113402.

[21] Hirsch A and Vostrowsky O 2005 Functionalization of Carbon Nanotubes Top Curr Chem 245, 193.

[22] Kürti J, Zolyomi V, Kertesz M and Sun G 2003 The geometry and the radial breathing mode of carbon nanotubes: beyond the ideal behaviour New J. Phys. 5, 125.

[23] Niyogi S, Hamon MA, Hu H, Zhao B, Bhowmik P, Sen R, Itkis ME and Haddon RC 2002 Chemistry of Single-Walled Carbon Nanotubes Acc. Chem. Res. 35, 1105.

[24] Saito R, Dresselhaus G and Dresselhaus MS 2000 Trigonal warping effect of carbon nanotubes Phys. Rev. B 61, 2981.

[25] Reich S and Thomsen C 2000 Chirality dependence of the density-of-states singularities in carbon nanotubes Phys. Rev. B 62, 4273.

[26] Kataura H, Kumazawa Y, Maniwa Y, Umezu I, Suzuki S, Ohtsuka Y and Achiba Y 1999 Optical properties of single-wall carbon nanotubes Synthetic Metals 103, 2555.

[27] The data for the generation of the Kataura plot are a courtesy of Prof. S. Maruyama and are publicly available at his web-page http://www.photon.t.utokyo.ac.jp/ maruyama/kataura/kataura.html.

[28] O’Connell MJ, Bachilo SM, Huffman CB, Moore WC, Strano MS, Haroz EH, Rialon KL, Boul PJ, Noon WH, Kittrell C, Ma J, Hauge RH, Weisman RW and Smalley RE 2002 Band Gap Fluorescence from Individual Single-Walled Carbon Nanotubes Science 297, 593.

[29] Lefebvre J, Homma Y and Finnie P 2003 Bright Band Gap Photoluminescence from Unprocessed Single-Walled Carbon Nanotubes Phys. Rev Lett. 90, 217401. 
[30] Oyama Y, Saito R, Sato K, Jiang J, Samsonidze GG, Grüneis A, Miyauchi Y, Maruyama S, Jorio A, Dresselhaus G and Dresselhaus M 2006 Photoluminescence intensity of single-wall carbon nanotubes Carbon 44, 873.

[31] Lefebvre J, Maruyama S and Finnie P 2008 Photoluminescence: Science and Applications Topics in Applied Physics 111, 287-319 from "Carbon Nanotubes: Advanced Topics in the Synthesis, Structure, Properties and Applications”, edited by A. Jorio et al. (Springer, Berlin, 2008).

[32] Thomsen C and Reich S 2007 Raman Scattering in Carbon Nanotubes Topics Appl. Physics 108, 115.

[33] Bachilo S, Strano M, Kittrell C, Hauge R, Smalley R and Weisman R 2002 StructureAssigned Optical Spectra of Single-Walled Carbon Nanotubes Science 298, 2361.

[34] Choi JH and Strano MS 2007 Solvatochromism in single-walled carbon nanotubes Appl. Phys. Lett. 90, 223114.

[35] Berger S, Iglesias F, Bonnet P, Voisin C, Cassabois G, Lauret JS, Delalande C and Roussignol P 2009 Optical properties of carbon nanotubes in a composite material: The role of dielectric screening and thermal expansion J. Appl. Phys. 105, 094323.

[36] Wang F, Dukovic G, Brus LE and Heinz TF 2005 The Optical Resonances in Carbon Nanotubes Arise from Excitons Science 308, 838.

[37] Maultzsch J, Pomraenke R, Reich S, Chang E, Prezzi D, Ruini A, Molinari E, Strano MS, Thomsen C and Lienau C 2005 Exciton binding energies in carbon nanotubes from two-photon photoluminescence Phys. Rev. B 72 241402(R).

[38] Maultzsch J, Pomraenke R, Reich S, Chang E, Prezzi D, Ruini A, Molinari E, Strano MS, Thomsen C and Lienau C 2006 Excitons in Carbon Nanotubes Phys. Stat. Sol. B 2433204.

[39] Malic E, Maultzsch J, Reich S and Knorr A 2010 Excitonic absorption spectra of metallic single-walled carbon nanotubes Phys. Rev. B 82, 035433.

[40] Cognet L, Tsyboulski DA, Rocha JDR, Doyle CD, Tour JM and Weisman RB 2007 Stepwise quenching of exciton fluorescence in carbon nanotubes by single-molecule reactions Science 316, 1465.

[41] Tasis D, Tagmatarchis N, Bianco B and Prato M 2006 Chemistry of Carbon Nanotubes Chem. Rev. 106, 1105.

[42] Cambré S, Santos SM, Wenseleers W, Nugraha ART, Saito R, Cognet L and Lounis B 2012 Luminescence Properties of Individual Empty and Water-Filled Single-Walled Carbon Nanotubes ACS Nano 6, 2649.

[43] Botka B, Füstös ME, Klupp G, Kocsis D, Székely E, Utczás M, Simándi B, Botos Á, Hackl R and Kamarás K 2012 Low-temperature encapsulation of coronene in carbon nanotubes Phys. Stat. Sol. B 249, 2432. 
[44] Chernov AI, Fedotov PV, Anoshkin IV, Nasibulin AG, Kauppinen EI, Kuznetsov VL and Obraztsova ED 2014 Single-walled carbon nanotubes as a template for coronene stack formation Phys. Stat. Sol. B 251, 2372.

[45] Gaufrès, E, Tang NY-Wa, Lapointe F, Cabana J, Nadon M-A, Cottenye N, Raymond F, Szkopek T and Martel R 2014 Giant Raman scattering from J-aggregated dyes inside carbon nanotubes for multispectral imaging Nat. Photonics 8, 72.

[46] Cambré S, Campo J, Beirnaert C, Verlackt C, Cool P and Wenseleers W 2015 Asymmetric dyes align inside carbon nanotubes to yield a large nonlinear optical response Nat. Nanotechnol. 10, 248.

[47] Pan XL, Fan ZL, Chen W, Ding YJ, Luo HY and Bao 2007 Enhanced ethanol production inside carbon-nanotube reactors containing catalytic particles Nat. Materials 6, 507.

[48] Chamberlain TW, Biskupek J, Rance GA, Chuvilin A, Alexander TJ, Bichoutskaia E, Kaiser U and Khlobystov AN 2012 Size, Structure, and Helical Twist of Graphene Nanoribbons Controlled by Confinement in Carbon Nanotubes ACS Nano 6, 3943.

[49] Laudenbach J, Schmid D, Herziger F, Hennrich F, Kappes M, Muoth M, Haluska M, Hof F, Backes C, Hauke F, Hirsch A and Maultzsch J 2017 Diameter dependence of the defect-induced Raman modes in functionalized carbon nanotubes Carbon 112, 1.

[50] Hof F, Bosch S, Englert JM, Hauke F and Hirsch A 2012 Statistical Raman Spectroscopy: A Method for the Characterization of Covalently Functionalized Single-Walled Carbon Nanotubes Angew. Chem. Int. Ed. 51, 11727.

[51] Holzinger M, Abraham J, Whelan P, Graupner R, Ley L, Hennrich F, Kappes M and Hirsch A 2003 Functionalization of Single-Walled Carbon Nanotubes with (R-)Oxycarbonyl Nitrenes J. Am. Chem. Soc. 125, 8566.

[52] Graupner R, Abraham J, Wunderlich D, Vencelova A, Lauffer P, Roehrl J, Hundhausen M, Ley L and Hirsch A 2006 Nucleophilic-Alkylation-Reoxidation: A Functionalization Sequence for Single-Wall Carbon Nanotubes J. Am. Chem. Soc. 128, 6683.

[53] Syrgiannis Z, Hauke F, Röhrl J, Hundhausen M, Graupner R, Elemes Y and Hirsch A 2008 Covalent Sidewall Functionalization of SWNTs by Nucleophilic Addition of Lithium Amides Eur. J. Org. Chem. 2008, 2544.

[54] Syrgiannis Z, Gebhardt B, Dotzer C, Hauke F, Graupner R and Hirsch A 2010 Reductive Retrofunctionalization of Single-Walled Carbon Nanotubes Angew. Chem., Int. Ed. 49, 3322.

[55] Gebhardt B, Syrgiannis Z, Backes C, Graupner R, Hauke F and Hirsch A 2011 Carbon Nanotube Sidewall Functionalization with Carbonyl Compounds-Modified Birch Conditions vs the Organometallic Reduction Approach J. Am. Chem. Soc. 133, 7985.

[56] Hof F, Bosch S, Eigler S, Hauke F and Hirsch 2013 New Basic Insight into Reductive Functionalization Sequences of Single Walled Carbon Nanotubes (SWCNTs) J. Am. Chem. Soc. 135, 18385. 
[57] Hof F, Schäfer RA, Weiss C, Hauke F and Hirsch A, 2014 Novel Lambda-3-Iodane Based Functionalization of Synthetic Carbon Allotropes (SCAs) - Common Concepts and Quantification of the Degree of Addition Chem. Eur. J. 20, 16644.

[58] Bosch S, Zeininger L, Hauke F and Hirsch A 2014 A Supramolecular Approach for the Facile Solubilization and Separation of Covalently Functionalized Single-Walled Carbon Nanotubes Chem. Eur. J. 20, 2537.

[59] Wunderlich D, Hauke F and Hirsch A 2008 Preferred functionalization of metallic and smalldiameter single walled carbon nanotubes via reductive alkylation J. Mater. Chem. 18, 1493.

[60] Wunderlich D, Hauke F and Hirsch A 2008 Preferred functionalization of metallic and smalldiameter single-walled carbon nanotubes by nucleophilic addition of organolithium and magnesium compounds followed by Reoxidation Chemistry--A European Journal 14, 1607.

[61] Gebhardt B, Graupner R, Hauke F and Hirsch A 2010 A Novel Diameter-Selective Functionalization of SWCNTs with Lithium Alkynylides Eur. J. Org. Chem. 2010, 1494.

[62] Gebhardt B, Hof F, Backes C, Mueller M, Plocke T, Maultzsch J, Thomsen C, Hauke F and Hirsch A 2011 Selective Polycarboxylation of Semiconducting Single-Walled Carbon Nanotubes by Reductive Sidewall Functionalization J. Am. Chem. Soc. 133, 19459.

[63] Knirsch KC, Hof F, Lloret V, Mundloch U, Hauke F and Hirsch A 2016 Topology-Driven Reductive Silylation of Synthetic Carbon Allotropes J. Am. Chem. Soc. 138, 15642.

[64] Gebhardt J, Bosch S, Hof F, Hauke F, Hirsch A and Gorling A 2017 Selective reduction of SWCNTs - concepts and insights Journal of Materials Chemistry C 5, 3937.

[65] Bekyarova E, Sarkar S, Wang F, Itkis ME, Kalinina I, Tian X and Haddon RC 2013 Effect of covalent chemistry on the electronic structure and properties of carbon nanotubes and graphene Acc. Chem. Res. 46, 65.

[66] Gordeev G, Setaro A, Glaeske M, Jürgensen S and Reich S 2016 Doping in covalently functionalized carbon nanotubes: A Raman scattering study Phys. Stat. Sol. B 253, 2461.

[67] Piao Y, Meany B, Powell LR, Valley N, Kwon H, Schatz GC and Wang YH 2013 Brightening of carbon nanotube photoluminescence through the incorporation of $\mathrm{sp}^{3}$ defects Nat. Chemistry $\mathbf{5}$, 840.

[68] Miyauchi Y, Iwamura M, Mouri S, Kawazoe T, Ohtsu M and Matsuda K 2013 Brightening of excitons in carbon nanotubes on dimensionality modification Nat. Photonics 7, 715.

[69] Hartmann NH, Velizhanin KA, Haroz EH, Kim M, Ma X, Wang YH, Htoon H and Doorn SK 2016 Photoluminescence Dynamics of Aryl sp ${ }^{3}$ Defect States in Single-Walled Carbon Nanotubes ACS Nano 10, 8355.

[70] Kalinina I, Bekyarova E, Sarkar S, Wang F, Itkis ME, Tian X, Niyogi S, Jha N and Haddon RC 2012 Hexahapto-Metal Complexes of Single-Walled Carbon Nanotubes Macromol. Chem. Phys. 213, 1001. 
[71] Brennan LC and Gun'ko Y 2015 Advances in the Organometallic Chemistry of Carbon Nanomaterials Organometallics 34, 2086.

[72] Tournus F, Latil S, Heggie MI and Charlier JC $2005 \square$ stacking interaction between carbon nanotubes and organic molecules Phys. Rev. B 72, 075431.

[73] Perez EM and Martín N $2015 \pi-\pi$ interactions in carbon nanostructures Chem. Soc. Rev. 44, 6425.

[74] O'Connell MJ, Boul P, Ericson LM, Huffman C, Wang Y, Haroz E, Kuper C, Tour J, Ausman KD and Smalley RE 2001 Reversible water-solubilization of single-walled carbon nanotubes by polymer wrapping Chem. Phys. Lett. 342, 265.

[75] Islam MF, Rojas E, Bergey DM, Johnson AT and Yodh AG 2003 High weight fraction surfactant solubilization of single-wall carbon nanotubes in water Nano Letters 3, 269.

[76] Ham HT, Choi YS and Chung IJ 2005 An explanation of dispersion states of single-walled carbon nanotubes in solvents and aqueous surfactant solutions using solubility parameters Journal of Colloid and Interface Science 286, 216.

[77] Vaisman L, Wagner HD and Marom G 2006 The role of surfactants in dispersion of carbon nanotubes Advances in Colloids and Interface Science 128-130, 37.

[78] Wenseleers W, Vlasov II, Goovaerts E, Obraztsova ED, Lobach AS and Bouwen A 2004 Efficient Isolation and Solubilization of Pristine Single-Walled Nanotubes in Bile Salt Micelles Adv. Funct. Mat. 14, 1105.

[79] Marquis R, Greco G, Sadokierska I, Lebedkin S, Kappes MM, Michel T, Alvarez L, Sauvajol JL, Meunier S and Mioskowski C 2008 Supramolecular Discrimination of Carbon Nanotubes According to Their Helicity Nano Lett. 8, 1830.

[80] Ju SY, Doll J, Sharma I and Papadimitrakopoulos F 2008 Selection of carbon nanotubes with specific chiralities using helical assemblies of flavin mononucleotide Nat. Nanotechnol. 3, 356.

[81] Setaro A, Popeney CS, Trappmann B, Datsyuk V, Haag R and Reich S 2010 Polyglycerolderived amphiphiles for single walled carbon nanotube suspension Chem. Phys. Lett. 493, 147.

[82] Setaro A, Popeney CS, Trappmann B, Haag R and Reich S 2010 Interaction between single walled carbon nanotubes and alkyl-polyglycerol derivatives Phys. Stat. Sol. B 247, 2758.

[83] Bluemmel B, Setaro A, Popeney CS, Trappmann B, Haag R and Reich S 2011 Amphiphile replacement on carbon nanotube surfaces: Effect of aromatic groups on the interaction strength Phys. Stat. Sol. B 248, 2532.

[84] Popeney CS, Setaro A, Mutihac RC, Bluemmel P, Trappmann B, Vonneman J, Reich S and Haag R 2012 Polyglycerol-Derived Amphiphiles for the Solubilization of Single-Walled Carbon Nanotubes in Water: A Structure-Property Study ChemPhysChem 13, 203.

[85] Ernst F, Heek T, Haag R, Reich S and Setaro A 2012 Chirally enhanced solubilization through perylene-based surfactant Phys. Status Solidi B 249, 2465. 
[86] Ernst F, Heeg S, Heek T, Setaro A, Haag R and Reich S 2013 Selective interaction between nanotubes and perylene based surfactant Phys. Status Solidi RRL 7, 546.

[87] Setaro A, Popeney CS, Witt MU, Bluemmel P, Glaeske M, Haag R and Reich S 2015 Chiral selectivity of polyglycerol-based amphiphiles incorporating different aromatic cores Phys. Stat. Sol. B 252, 2536.

[88] Setaro A, Bluemmel P, Witt MU, Narula R and Reich S 2016 Carbon Nanotube Chirality Enrichment through Chirality-Selective Precipitation Phys. Stat. Sol. B 253, 2380.

[89] Tu X, Manohar S, Jagota A and Zheng M 2009 DNA sequence motifs for structure-specific recognition and separation of carbon nanotubes Nature 460, 250.

[90] Gomulya W, Costanzo GD, Figueiredo de Carvalho EJ, Bisri SZ, Derenskyi V, Fritsch M, Fröhlich N, Allard S, Gordiichuk P, Herrmann A, Marrink SJ, dos Santos MC, Scherf U and Loi MA 2013 Semiconducting Single-Walled Carbon Nanotubes on Demand by Polymer Wrapping Adv. Mat. 25, 2948.

[91] Ozawa H, Fujigaya T, Song S.et al. 2011 Different Chiral Selective Recognition/Extraction of $(\mathrm{n}, \mathrm{m})$ Single-walled Carbon Nanotubes Using Copolymers Carrying a Carbazole or Fluorene Moiety Chem. Lett. 40, 470.

[92] Chen RJ, Zhang Y, Wang D and Dai H 2001 Noncovalent Sidewall Functionalization of Single-Walled Carbon Nanotubes for Protein Immobilization J. Am. Chem. Soc. 123, 3838.

[93] Kruss S, Salem DP, Vuković L, Lima B, Vander Ende E, Boyden ES and Strano MS 2017 High-resolution imaging of cellular dopamine efflux using a fluorescent nanosensor array PNAS 114, 1789.

[94] Kruss S, Hilmer AJ, Zhang J, Reuel NF, Mu B and Strano MS 2013 Carbon nanotubes as optical biomedical sensors Adv. Drug Del. Rev. 65, 1933.

[95] Sachez-Valencia JR, Dienel T, Groning O, Shorubalko I, Mueller A, Jansen M, Amsharov K, Ruffieux P and Fasel R 2014 Controlled synthesis of single-chirality carbon nanotubes Nature 512, 61.

[96] Arnold MS, Green AA, Hulvat JF, Stupp SI and Hersam MC, 2006 Sorting carbon nanotubes by electronic structure using density differentiation Nat. Nanotechnol. 1, 60.

[97] Krupke R, Hennrich F, von Loehneysen H and Kappes MM 2003 Separation of Metallic from Semiconducting Single-Walled Carbon Nanotubes Science 301, 344.

[98] Liu H, Nishide D, Tanaka T and H. Kataura H 2011Large-scale single-chirality separation of single-wall carbon nanotubes by simple gel chromatography Nat. Commun. 2, 309.

[99] Gui H, Chen H, Khripin CY, Liu B, Fagan JA, Zhou C and Zheng M 2016 A facile and lowcost length sorting of single-wall carbon nanotubes by precipitation and applications for thin-film transistors Nanoscale 8, 3467.

[100] Ao G, Streit JK, Fagan JA and Zheng M 2016 Differentiating Left- and Right-Handed Carbon Nanotubes by DNA J. Am. Chem. Soc. 138, 16677. 
[101] Khripin CY, Fagan JA and Zheng M 2013 Spontaneous Partition of Carbon Nanotubes in Polymer-Modified Aqueous Phases J. Am. Chem. Soc 135, 6822.

[102] Subbaiyan NK, Parra-Vasquez ANG, Cambré S, Cordoba MAS, Yalcin SE, Hamilton CE, Mack NH, Blackburn JL, Doorn SK and Duque JG 2015 Bench-top aqueous two-phase extraction of isolated individual single-walled carbon nanotubes Nano Res. 8, 1755.

[103] Zheng M 2017 Sorting Carbon Nanotubes Top Curr Chem 375, 13.

[104] Backes C, Hauke F, Schmidt CD and Hirsch A 2009 Fractioning HiPco and CoMoCAT SWCNTs via density gradient ultracentrifugation by the aid of a novel perylene bisimide derivative surfactant Chem. Commun. 19, 2643.

[105] Backes C, Schmidt CD, Hauke F, Boettcher C and Hirsch A 2009 High Population of Individualized SWCNTs through the Adsorption of Water-Soluble Perylenes J. Am. Chem. Soc. 131, 2172.

[106] Ehli C, Oelsner C, Guldi DM, Mateo-Alonso A, Prato M, Schmidt C, Backes C, Hauke F and Hirsch A 2009 Manipulating single-wall carbon nanotubes by chemical doping and charge transfer with perylene dyes Nat. Chem. 1, 243.

[107] Backes C, Schmidt CD, Rosenlehner K, Hauke F, Coleman JN and Hirsch A 2010 Nanotube Surfactant Design: The Versatility of Water-Soluble Perylene Bisimides Adv. Mater. 22, 788.

[108] Backes C, Englert JM, Bernhard N, Hauke F and Hirsch A 2010 Optical Visualization of Carbon Nanotubes - a Unifying Linkage Between Microscopic and Spectroscopic Characterization Techniques Small 6, 1968.

[109] Backes C, Mundloch U, A. Ebel, Hauke F and Hirsch A 2010 Dispersion of HiPco ${ }^{\circledR}$ and CoMoCAT ${ }^{\circledR}$ Single-Walled Nanotubes (SWNTs) by Water Soluble Pyrene Derivatives Depletion of Small Diameter SWNTs Chem. Eur. J. 16, 3314.

[110] Backes C, Mundloch U, Schmidt CD, Coleman JN, Wohlleben W, Hauke F and Hirsch A 2010 Enhanced Adsorption Affinity of Anionic Perylene-Based Surfactants towards SmallerDiameter SWCNTs Chem. Eur. J. 16, 13185.

[111] Backes C, Hauke F and Hirsch A 2011 The Potential of Perylene Bisimide Derivatives for the Solubilization of Carbon Nanotubes and Graphene Adv. Mater. 23, 2588.

[112] Backes C, Bosch S, Mundloch U, Hauke F and Hirsch A 2011 Density Gradient Ultracentrifugation on Carbon Nanotubes According to Structural Integrity as a Foundation for an Absolute Purity Evaluation ChemPhysChem 12, 2576.

[113] Backes C, Schunk T, Hauke F and Hirsch A 2011 Counterion effect on the aggregation of anionic perylene dyes and the influence on carbon nanotube dispersion efficiencies J. Mater. Chem. 21, 3554.

[114] Backes C, Hauke F and Hirsch A 2013 Tuning the adsorption of perylene-based surfactants on the surface of single-walled carbon nanotubes Phys. Stat. Sol. B 250, 2592. 
[115] Ernst F, Heek T, Setaro A, Haag R and Reich S 2012 Energy transfer in nanotube-perylene complexes Adv. Funct. Mater. 22, 3921.

[116] Ernst F, Heek T, Setaro A, Haag R and Reich S 2013 Functional Surfactants for Carbon Nanotubes: Effects of Design J. Phys. Chem. C 117, 1157.

[117] Ernst F, Heek T, Setaro A, Haag R and Reich S 2013 Excitation characteristics of different energy transfer in nanotube-perylene complexes Appl. Phys. Lett. 102, 233105.

[118] Roquelet C., Lauret JS, Alain-Rizzo V, Voisin C, Fleurier R, Delarue M, Garrot D, Loiseau A, Roussignol P, Delaire JA and Deleporte E 2010 П-Stacking Functionalization of Carbon Nanotubes through Micelle Swelling ChemPhysChem 11, 1667.

[119] Roquelet C, Garrot D, Lauret JS, Voisin C, Alain-Rizzo V, Roussignol Ph, Delaire JA and Deleporte E 2010 Quantum efficiency of energy transfer in noncovalent carbon nanotube/porphyrin compounds Appl. Phys. Lett. 97, 141918.

[120] Garrot D, Langlois B, Roquelet C, Michel T, Roussignol P, Delalande C, Deleporte E, Lauret JS and Voisin C 2011 Time-Resolved Investigation of Excitation Energy Transfer in Carbon Nanotube-Porphyrin Compounds J. Phys. Chem. C 115, 23283.

[121] Roquelet C, Vialla F, Diederichs C, Roussignol Ph, Delalande C,Deleporte E, Lauret JS and Voisin 2012 Local Field Effects in the Energy Transfer between a Chromophore and a Carbon Nanotube: A Single-Nanocompound Investigation ACS Nano 6, 8796.

[122] Roquelet C, Langlois B, Vialla F, Garrot D, Lauret JS and Voisin C 2012 Light harvesting with non covalent carbon nanotube/porphyrin compounds Chem. Phys. 413, 45.

[123] Wang RK, Chen WC, Campos DK and Ziegler KJ 2008 Swelling the micelle core surrounding single-walled carbon nanotubes with water-immiscible organic solvents. Journ. Am. Chem. Soc. 130, 16330.

[124] Clave G, Delport, G, Roquelet C, Lauret JS, Deleporte E, Vialla F, Langlois B, Parret, R, Voisin, C, Roussignol, Ph, Jousselme B, Gloter A, Stephan O, Filoramo A, Derycke V and Campidelli S 2013 Functionalization of Carbon Nanotubes through Polymerization in Micelles: A Bridge between the Covalent and Noncovalent Methods Chem. Mat. 25, 2700.

[125] Vialla F, Delport G, Chassagneux Y, Roussignol Ph, Lauret JS and Voisin C 2016 Diameterselective non-covalent functionalization of carbon nanotubes with porphyrin monomers Nanoscale 8, 2326.

[126] Delport G, Orcin-Chaix L, Campidelli S, Voisin C and Lauret JS 2017 Controlling the kinetics of the non-covalent functionalization of carbon nanotubes using sub-cmc dilutions in a cosurfactant environment Nanoscale 9, 2646.

[127] Setaro A, Adeli M, Glaeske M, Przyrembel D, Bisswanger T, Gordeev G, Maschietto F, Faghani A, Paulus B, Weinelt M, Arenal R, Haag R and Reich S 2017 Preserving $\pi$-conjugation in covalently functionalized carbon nanotubes for optoelectronic applications Nat. Comm. 8, 14281. 
[128] Kumar I, Rana S and Cho JW 2011 Cycloaddition reactions: a controlled approach for carbon nanotube functionalization Chem. Eur. J. 17, 11092.

[129] Chen Z, Nagase S, Hirsch A, Haddon RC, Thiel W and von Ragué Schleyer P 2004 Side-wall opening of single-walled carbon nanotubes (SWCNTs) by chemical modification: a critical theoretical study Angew. Chem. Int. Ed. 43, 1552.

[130] Lee YS and Marzari N 2006 Cycloaddition functionalizations to preserve or control the conductance of carbon nanotubes Phys. Rev. Lett. 97, 116801.

[131] Li J, Jia G, Zhang Y and Chen Y 2006 Bond-Curvature effect of sidewall [2.1] cycloadditions of single-walled carbon nanotubes: a new criterion to the adduct structures Chem. Mater. 18, 3579.

[132] Zhang K, Zhang Q, Liu C, Marzari N and Stellacci F 2012 Diameter effect on the sidewall functionalization of single-walled carbon nanotubes by addition of dichlorocarbene Adv. Funct. Mater. 22, 5216.

[133] Brown GH 1971 Photochromism (New York: Wiley).

[134] Crano JC and Guglielmetti RJ 1999 Organic Photochromic and Thermochromic Compounds (New York: Kluwer Academic Press).

[135] Feringa BL 2001Molecular Switches (Weinheim: Wiley).

[136] Dürr H and Bouas-Laurent H 2003 Photochromism: Molecules and Systems (Amsterdam: Elsevier).

[137] Natali M and Giordani S 2012 Molecular switches as photocontrollable "smart” receptors Chem. Soc. Rev. 41, 4010.

[138] Fischer E and Hirshberg Y 1952 Formation of coloured forms of spirans by low-temperature irradiation Journal of the Chemical Society 4522-4524.

[139] Raymo FM, Alvarado RJ, Giordani S and Cejas MA 2003 Memory Effects Based on Intermolecular Photoinduced Proton Transfer JACS 125, 2361.

[140] Seefeldt B, Kasper R, Beining M, Mattay J, Arden-Jacob J, Kemnitzer N, Drexhage KH, Heilemann M and Sauer M 2010 Spiropyrans as molecular optical switches Photochem. Photobiol. Sci. 9, 213.

[141] Matsuzawa Y, Kato H, Ohyama H, Nishide D, Kataura H and Yoshida M 2011 Photoinduced Dispersibility Tuning of Carbon Nanotubes by a Water-Soluble Stilbene as a Dispersant Adv. Mat. 23, 3922.

[142] Kördel C, Setaro A, Bluemmel P, Popeney CS, Reich S and Haag R 2012 Controlled reversible debundling of single-walled carbon nanotubes by photo-switchable dendritic surfactants Nanoscale 4, 3029.

[143] Bluemmel P, Setaro A, Popeney CS, Haag R and Reich S 2010 Dispersion of Carbon Nanotubes using an Azobenzene Derivative Phys. Status Solidi B 248, 2532. 
[144] Zhou X, Zifer T, Wong BM, Krafcik KL, Léonard F and Vance AL 2009 Color Detection Using Chromophore-Nanotube Hybrid Devices Nano Lett. 9, 1028.

[145] Setaro A, Kreft SK, Åxman Petersen M, Brøndsted Nielsen M and Reich S 2014 Optical properties of carbon nanotubes coated with orthogonal dipole switches Phys. Status Solidi B 251, 2356.

[146] Kreft SK, Åxman Petersen M, Brøndsted Nielsen M, Reich S and Setaro A 2015 Isomerization of orthogonal molecular switches encapsulated within micelles solubilizing carbon nanotubes J. Phys. Chem. C 119, 15731.

[147] Klajn R 2014 Spiropyran-based dynamic materials Chem. Soc. Rev. 43, 184.

[148] Khairutdinov RF, Itkis ME and Haddon RC 2004 Microscopic Model of the Optical Absorption of Carbon Nanotubes Functionalized with Molecular Spiropyran Photoswitches 4, 1529.

[149] Guo XF, Huang LM, O’Brien S, Kim P and Nuckolls C 2005 Directing and Sensing Changes in Molecular Conformation on Individual Carbon Nanotube Field Effect Transistors J. Am. Chem. Soc. 127(43) 15045-15047

[150] Del Canto E, Flavin K, Natali M, Perova T and Giordani S 2010 Functionalization of singlewalled carbon nanotubes with optically switchable spiropyrans Carbon 48, 2815.

[151] Del Canto E, Natali M, Movia D and Giordani S 2012 Photo-controlled release of zinc metal ions by spiropyran receptors anchored to single-walled carbon nanotubes Phys. Chem. Chem. Phys. 14, 6034.

[152] Setaro A, Bluemmel P, Maity C, Hecht S and Reich S 2012 Non-Covalent Functionalization of Individual Nanotubes with Spiropyran-Based Molecular Switches Adv. Funct. Mater. 22, 2425.

[153] Malic E, Weber C, Richter M, Atalla V, Klamroth T, Saalfrank P, Reich S and Knorr A 2011 Microscopic Model of the Optical Absorption of Carbon Nanotubes Functionalized with Molecular Spiropyran Photoswitches Phys. Rev. Lett. 106, 097401.

[154] Malic E, Setaro A, Bluemmel P, Sanz-Navarro CF, Ordejon P, Reich S and Knorr A 2012 Carbon nanotubes as substrates for molecular spiropyran-based switches J. Phys. Cond. Matt. 24, 394006.

[155] Bluemmel P, Setaro A, Maity C, Hecht S and Reich S 2012 Designing a spiropyran-based molecular switch for carbon nanotube functionalization: Influence of anchor groups and tubeswitch separation Phys. Status Solidi B 249, 2479.

[156] Bluemmel P, Setaro A, Maity C, Hecht S and Reich S 2012 Tuning the interaction between carbon nanotubes and dipole switches: influence of the change of the nanotube-spiropyran distance J. Phys. Cond. Matt. 24, 394005.

[157] Glaeske M, Bluemmel P, Jürgensen S, Setaro A and Reich S 2017 Dipole-Switch Induced Modification of the Emissive Response of Carbon Nanotubes, submitted to JPCM. 
[158] Jones M, Engtrakul C, Metzger WK, Ellingson RJ, Nozik AJ, Heben MJ and Rumbles G 2005 Analysis of photoluminescence from solubilized single-walled carbon nanotubes Phys. Rev. B 71, 115426.

[159] Carlson LJ, Maccagnano SE, Zheng M, Silcox J and Krauss TD 2007 Fluorescence efficiency of individual carbon nanotubes Nano Lett. 7, 3698.

[160] Ju S, Kopcha W and Papadimitrakopoulos F 2009 Brightly fluorescent single-walled carbon nanotubes via an oxygen excluding surfactant organization Science 323, 1319.

[161] Parbeinos V, Tersof J and Avouris P 2005 Radiative lifetime of excitons in SWNTs Nano Lett. 5, 2495.

[162] Xia F, Steiner M, Lin YM and Avouris P 2008 A microcavity controlled, current-driven, onchip nanotube emitter at infrared wavelengths Nat. Nanotechnol. 3, 609.

[163] Gaufrès E, Izard N, Roux XL, Kazaoui S, Marris-Morini D, Cassan E and Vivien L 2010 Optical microcavity with semiconducting single-wall carbon nanotubes Opt. Express 18, 5740.

[164] Watahiki R, Shimada T, Zhao P, Chiashi S, Iwamoto S, Arakawa Y, Maruyama S and Kato YK 2012 Enhancement of carbon nanotube photoluminescence by photonic crystal nanocavities Appl. Phys. Lett. 101, 141124.

[165] Maier SA 2007 Plasmonics: Fundamentals and Applications (Springer US).

[166] Heeg S, Oikonomou A, Fernandez-Garcia R, Lehmann C, Maier SA, Vijayaraghavan A and Reich S 2014 Plasmon-Enhanced Raman Scattering by Carbon Nanotubes Optically Coupled with Near-Field Cavities Nano Lett. 14, 1762.

[167] Hong G, Tabakman SM, Welsher K, Wang H, Wang X, Dai H, Matsuda K, Kanemitsu Y, Irie K, Saiki T, Someya T, Miyauchi Y and Maruyama, S 2010 Metal-enhanced fluorescence of carbon nanotubes J. Am. Chem. Soc. 132, 15920.

[168] Sakashita T, Miyauchi Y, Matsuda K and Kanemitsu Y 2010 Plasmon-assisted photoluminescence enhancement of single walled carbon nanotubes on metal surfaces Appl. Phys. Lett. 97, 063110.

[169] Zakharko Y, Graf A and Zaumseil J. 2016 Plasmonic Crystals for Strong Light-Matter Coupling in Carbon Nanotubes Nano Lett. 16, 6504.

[170] Graf A, Tropf L, Zakharko Y, Zaumseil J and Gather MC 2016 Near-infrared excitonpolaritons in strongly coupled single-walled carbon nanotube microcavities Nat. Comm. 7, 13078.

[171] Zhang JJ, Gu MM, Zheng TT and Zhu JJ 2009 Synthesis of Gelatin-Stabilized Gold Nanoparticles and Assembly of Carboxylic Single-Walled Carbon Nanotubes/Au Composites for Cytosensing and Drug Uptake Anal. Chem. 81, 6641.

[172] Motl NE, Smith AF, De Santis CJ and Skrabalak SE 2014 Engineering plasmonic metal colloids through composition and structural design Chem. Soc. Rev. 43, 3823.

[173] Lakowicz J R 2006 Principles of Fluorescence (Berlin: Springer). 
[174] Glaeske M and Setaro A 2013 Nanoplasmonic Colloidal Suspensions for the Enhancement of the Single Walled Carbon Nanotubes Luminescent Emission Nano Research 6, 593.

[175] Glaeske M and Setaro A 2014 Effect of hybrid isolation on the luminescence enhancement of carbon nanotube-gold nanorod composites Phys. Stat. Sol. B 251, 2480.

[176] Glaeske M, Kumar M, Bisswanger T, Vaitiekienas S, Soci C, Narula R, Bruno A and Setaro A 2017 Relaxation lifetimes of plasmonically enhanced hybrid gold - carbon nanotubes systems Nanotechnology 28, 255202.

[177] Zuloaga J and Nordlander P 2011 On the Energy Shift between Near-Field and Far-Field Peak Intensities in Localized Plasmon Systems Nano Lett. 11, 1280.

[178] Anger P, Bharadwaj P and Novotny L 2006 Enhancement and Quenching of Single-Molecule Fluorescence Phys. Rev. Lett. 96, 113002. 